\title{
Relationship between the Pi2 pulsations and the localized impulsive current associated with the current disruption in the magnetosphere
}

\author{
S. Fujita ${ }^{1,2}$, M. Itonaga ${ }^{3}$, and H. Nakata ${ }^{2}$ \\ ${ }^{1}$ Meteorological College, Asahi 7-4-81, Kashiwa, Chiba 277-0852, Japan \\ ${ }^{2}$ Department of Geophysics, Kyoto University, Kyoto 606-8502, Japan \\ ${ }^{3}$ Faculty of Education, Yamaguchi University, Yoshida 1677-1, Yamaguchi, Yamaguchi 753-8513, Japan
}

(Received September 14, 1999; Revised February 16, 2000; Accepted February 29, 2000)

\begin{abstract}
Behavior of fast-magnetosonic-mode MHD signals in the inner magnetosphere that are driven by the impulsive eastward current is investigated as a model of the Pi2 signal at midnight. The magnetosphere is treated as an axisymmetric cold MHD regime with dipole magnetic fields and has the plasmaspheric structure of the Alfvén speed distribution. MHD perturbation is assumed to be axisymmetric. Numerical calculation revealed the following: 1) The impulsive current induces the plasmasphere virtual resonance oscillation; 2) The compressional magnetic field perturbation is confined near the equator; 3) The waveform of the compressional magnetic perturbation in the plasmasphere depends on the spatial extent of the source current, its temporal variation, as well as its location; 4) The typical Pi2 waveform in the plasmasphere is obtained when the source current is located near the plasmapause $(L \leq 10)$; 5) When the source current is not located on the equator, the compressional component and the poloidal component have different waveforms.
\end{abstract}

\section{Introduction}

$\mathrm{Pi} 2$ pulsations are generally thought to be impulsively generated in association with the sudden change of the magnetic field in the near-Earth magnetotail from a tail-like configuration to a dipolar configuration (e.g., Yumoto, 1990). Recently, the propagation and generation mechanism of the $\mathrm{Pi} 2$ pulsations in middle and low latitudes and in the inner magnetosphere has been studied extensively via observational methods (Yeoman and Orr, 1989; Sutcliffe and Yumoto, 1989, 1991; Yumoto, 1990; Yumoto et al., 1989, 1990; Takahashi, 1994; Takahashi et al., 1992, 1995, 1999; Osaki et al., 1998). In response to these observations, theoretical/numerical studies on the behavior of low-latitude MHD waves have been carried out (Allan et al., 1986; Lee, 1996, 1998; Pekrides et al., 1997; Itonaga et al., 1997a,b; Lee and Kim, 1999).

Yeoman and Orr (1989) manifested from mid-latitude Pi2 observations that a $180^{\circ}$ phase shift of the $\mathrm{H}$ component is associated with the plasmapause latitude. Sutcliffe and Yumoto $(1989,1991)$ revealed that spectra of low-latitude pulsations are almost identical at different local times. These findings suggest that the behavior of the $\mathrm{Pi} 2$ pulsations in middle and low latitudes is explained by the "global mode" hypothesis presented by Kivelson and Southwood (1986). Yumoto (1990) suggested that middle- and low-latitude Pi2 pulsations are "global mode" trapped within the inner part of the magnetosphere. This idea was confirmed directly by ground-satellite coordinated observations of $\mathrm{Pi} 2$ pulsations by Takahashi et al. (1992, 1995). Takahashi et al. (1992)

Copy right (c) The Society of Geomagnetism and Earth, Planetary and Space Sciences (SGEPSS); The Seismological Society of Japan; The Volcanological Society of Japan; The Geodetic Society of Japan; The Japanese Society for Planetary Sciences. revealed a $180^{\circ}$ phase difference between the magnetic variations within the plasmasphere $\left(B_{H}\right.$ of Kakioka, $\left.L=1.2\right)$ and those beyond the plasmapause $\left(b_{z}\right.$, compressional component, of AMPTE CCE, $L>3$ ). This feature directly supports the "plasmasphere cavity-mode resonance" of Pi2 pulsations. Takahashi et al. (1995) analyzed the AMPTE CCE data and investigated the behavior of the Pi2 pulsation in the inner magnetosphere $(L<6)$ in detail and found that the "plasmasphere cavity-mode resonance" is associated with magnetospheric $\mathrm{Pi} 2$ pulsation. They also reported, based on AMPTE CCE data, that the compressional component of the Pi2 pulsation is confined near the magnetospheric equator in the plasmasphere. AMPTE CCE data covers the region of the magnetic latitude $\left(\lambda_{m}\right)$ smaller than $16^{\circ}$. Using the AKEBONO satellite data covering $\lambda_{m}$ larger than $20^{\circ}$, Osaki et al. (1998) found that the compressional component is not detected in the off-equatorial region. Therefore, the observations suggested that the current disruption in the near-Earth magnetotail generates low-latitude $\mathrm{Pi} 2$ pulsations, which show the "plasmasphere cavity-mode resonance" with the compressional component strongly confined near the magnetospheric equator. Now, a theoretical model corresponding to this observation is needed.

Before reviewing previous theoretical studies, it is important to clarify terms such as the "global mode" concerning the behavior of the MHD waves in the magnetosphere. Lee and Kim (1999) presented very useful illustrations that distinguish four types of behaviors of waves propagating in a non-uniform medium. The first is the "bounded wave" corresponding to the cavity/waveguide modes. Energy of this wave is perfectly trapped in the "well" (depression) of the Alfvén speed profile. The "global mode" belongs to this 
category. The second type of behavior is "tunneling" and "scattering" of the wave. This occurs when there is an enhancement in the Alfvén speed profile. When the wave becomes evanescent/propagating in this region, the situation is referred to as "tunneling"/"scattering". The third type of behavior is the "virtual trapped resonance" in which the Alfvén speed profile is increasing monotonically in one side and has an enhancement in the other side. In "virtual trapped resonance", the wave is partially trapped in the "well" between the wall and is the enhanced $V_{A}$ region and evanescent in the enhanced $V_{A}$ region. When the wave is not evanescent in the enhancement region, this is referred to as "virtual scattering resonance". Energy transport across the enhanced $V_{A}$ region occurs. This is different form the "bounded wave". "Virtual trapped resonance" and "virtual resonance scattering" indicate MHD waves propagating from the nightside magnetosphere to the plasmasphere. Therefore, the "plasmasphere cavity-mode resonance" of the low-latitude Pi2 pulsation should be referred to "plasmasphere virtual resonance". This term is used hereafter in the present paper.

Now, let us review previous theoretical and numerical studies. In order to examine the plasmasphere virtual resonance, several theoretical studies have investigated the magnetospheric response of the MHD impulse (Allan et al., 1986; Lee, 1996, 1998; Pekrides et al., 1997; Itonaga et al., 1997a,b; Lee and Kim, 1999). Pekrides et al. (1997) presented a 3D numerical simulation of MHD wave propagation in a cylindrical magnetosphere model with the plasmasphere structure. They obtained the plasmasphere virtual resonance oscillation, as well as the magnetosphere bounded mode oscillation. Their simulation includes the magnetosphere bounded mode because the magnetopause is assumed as a rigid boundary. Itonaga et al. (1997a,b) studied the 1D magnetospheric response of the impulse in the simplified magnetosphere model with plasmaspheric structure using a numerical Laplace transform technique. They assumed no outer boundary of the system because the nightside magnetosphere does not have a boundary such the magnetopause. They revealed that the magnetospheric signal is composed of the initial impulse and the normal modes in the system (the poles of the Laplace-transformed MHD equation). Lee (1998) and Lee and Kim (1999), on the other hand, presented a 2D analysis of the MHD signal induced by an impulse based on a simplified box-type magnetosphere-plasmasphere model. They insisted that instead of the ionosphere, a turning point in the inner magnetosphere acts as a wave reflection layer in the real magnetosphere.

All previous theoretical/numerical studies assumed the initial driving perturbation as the externally-charged electric field at the magnetopause boundary (Pekrides et al., 1997) or a wave impinging on the plasmasphere (Itonaga et al., 1997a,b; Lee, 1998; Lee and Kim, 1999). Therefore, the current disruption model (e.g., Yumoto, 1990) has not been explicitly treated in previous numerical/theoretical studies of the Pi2 pulsation. Moreover, the studies cited above use an unrealistic model. Only Lee (1996) uses the dipole magnetosphere with the plasmasphere. However, he presented only the wave characteristics in the stationary state sufficiently after the initial impulse. This treatment is not adequate for the simulation of the $\mathrm{Pi} 2$ pulsations. Therefore, we need to study the transient stage of the MHD signals generated by the impulsive current based on the dipole magnetosphere with the plasmasphere.

In addition to the $\mathrm{Pi} 2$ pulsation, the Psc pulsation is another phenomenon generated by the impulse (solar wind shock). Ground-based observation of the Psc pulsation revealed that the character of the Psc pulsation is essentially similar to that of the Pi2 pulsation in lower latitudes (Yumoto et al., 1994, 1997). Instead of the current disruption process, the enhanced Chapman-Ferraro current is used to generate the Psc pulsation.

The present paper intends to reveal the propagation character of the fast mode wave in the midnight meridian induced by the impulsive eastward (dusk-to-dawn) magnetospheric current based on the dipole magnetosphere model. The eastward current is the magnetospheric part of the current wedge that is believed to generate the $\mathrm{Pi} 2$ pulsation (Samson, 1982). In the present paper, this impulsive current is referred to as the source current. The source current is assumed to be localized in the field-aligned and radial directions. Since the present study represents the first attempt to characterize the behavior of fast-magnetosonic-mode MHD signals in the inner magnetosphere that are driven by the impulsive eastward current, for the purpose of simplification, the source current is assumed to be azimuthally uniform. The present paper is organized as follows. The second section describes the model employed and the numerical technique that is suitable for numerical analysis of the MHD wave propagation in the inner magnetosphere. The third section discusses the numerical results with an emphasis on the relationship between the waveform of the plasmasphere virtual resonance oscillation and the source current. The forth section describes the validity of the present numerical results and summarizes the main results.

\section{Model and Basic Equations}

In the present paper, MHD signal is assumed to be driven by the eastward current localized in the field-aligned and radial directions. In addition, the cold plasma model is employed.

\subsection{Model}

The model is the dipole magnetosphere having Alfvén speed $\left(V_{A}\right)$ that is a spatially non-uniform distribution having plasmaspheric structure. See Fujita and Glassmeier (1995) for the $V_{A}$ model. Here, only the radial variation of $V_{A}$ at the magnetospheric equator is shown in Fig. 1, in which the plasmapause structure of the $V_{A}$ profile is evident. In the present model, equatorial $V_{A}$ at $L=10$ is set as $500 \mathrm{~km} / \mathrm{sec}$. The magnetosphere is bounded by the ionosphere. Based on Itonaga et al. (1997b), the fast mode wave is reflected effectively by the solid Earth rather than the ionosphere. Bearing in mind that the distance between the solid Earth and the ionosphere is much smaller than the typical wavelength of the fast mode wave, it can be assumed that the boundary condition of the foot point of the field line for the fast mode wave is a perfect conductor. Figure 2 depicts the dipole magnetosphere model using the mesh configuration for the numerical calculation. In this figure, the $x$ and $z$ coordinates are employed, where $z$ is the axis of the south-to-north direction and $x$ is vertical to $z$. The magnetic field is assumed to be 


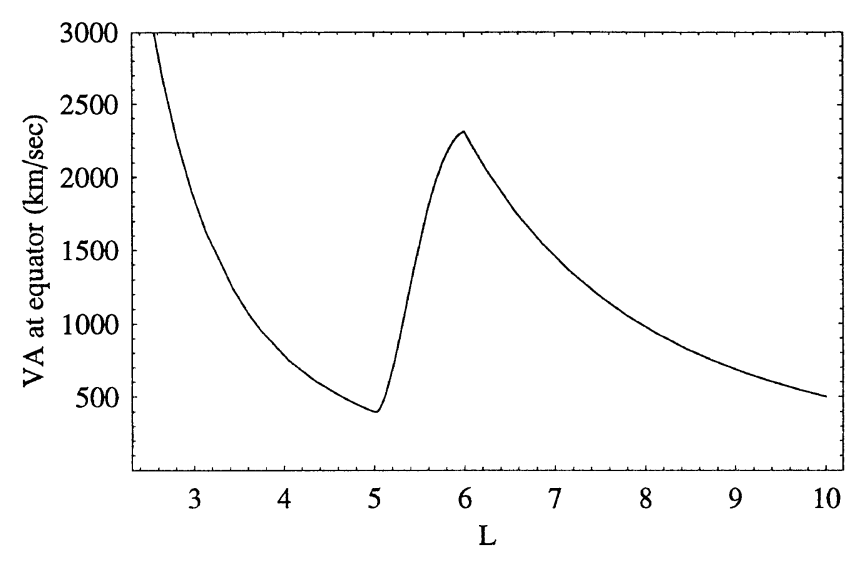

Fig. 1. Equatorial profile of $V_{A}$ in $L=3 \sim 10 . V_{A}$ at $L=10$ is 500 $\mathrm{km} / \mathrm{sec} . V_{A}$ is enhanced in the plasmapause $(L \simeq 6)$.

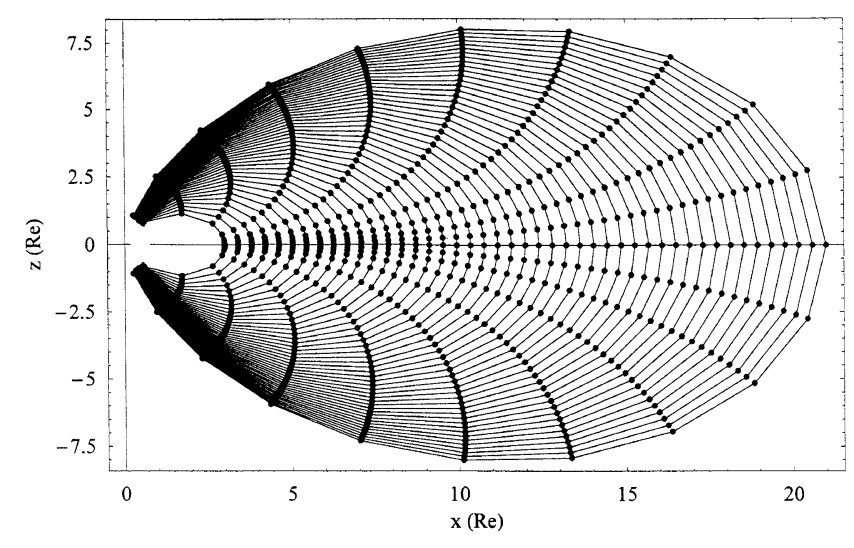

Fig. 2. The 2D model of the magnetosphere. This model is bounded by the inner boundary at $L=L_{i n}=3$ and by the outer boundary at $L=L_{\text {out }}=21$. The foot points of the field line are located at the ionospheres in both hemispheres. Dots in the figure denote the nodes used for the discrete numerical calculation. $z$ is the coordinate from the south pole to the north pole and $x$ is perpendicular to $z$.

vertical to the ionosphere. This assumption is valid in the region of $L>3$.

The coordinate system employed in the present paper is the dipole coordinate system, in which $\hat{e}$ and $\hat{n}$ are field-aligned and radially inward. Therefore, $\hat{\varphi}=\hat{e} \times \hat{n}$ is westward. The directions of $\hat{n}$ and $\hat{\varphi}$ are anti-parallel to those frequently used in other studies.

As for the boundary condition of MHD wave equations, the inner and outer boundaries ( $L=L_{\text {in }}$ and $L_{\text {out }}$, respectively) are assumed to be perfect conductors. The inner and outer boundaries are located at $L_{i n}=3$ and $L_{\text {out }}=21$, respectively. Since the ionosphere and the magnetic field is vertical in the present model, we assign the inner boundary at $L=3$. (There is no difficulty involved when the inner boundary is assigned at $L<3$. However, the oblique magnetic field to the ionosphere requires tedious treatment.) In order to model propagation of the MHD signal, it is important to take into account that the nightside magnetosphere has no outer boundary. Therefore, the outer boundary is located far from the position of the source current $(L \sim 10)$ in order to avoid the boundary effect from the behavior of the plasmasphere virtual resonance oscillation. In addition, we assign a special layer, in which the wave energy is absorbed in the outer part of the magnetosphere $\left(L=L^{\prime}>18\right)$. This layer has an artificial conductivity $\left(\sigma(L)=\sigma_{0}\left(L-L^{\prime}\right) /\left(L_{\text {out }}-L^{\prime}\right)\right)$. $\sigma_{0}=5 \times 10^{-3} \mathrm{mho} / \mathrm{m}$ is used in the present paper. However, in the present model, the wave-absorbing layer does not affect the behavior of the plasmasphere virtual resonance oscillation because the outer boundary is located far from the plasmasphere. Technically, this wave absorber stabilizes the numerical calculation.

\subsection{Source current}

The magnetospheric source current $\boldsymbol{J}(\boldsymbol{r}, t)$ ( $\boldsymbol{r}$ and $t$ are position and time, respectively) is assumed as $\mu_{0} \partial \boldsymbol{J} / \partial t=$ $\mu_{0} \hat{\varphi} \partial J_{\varphi} \partial / \partial t=\hat{\varphi} S_{\varphi}(\boldsymbol{r}) T(t)$, where $\mu_{0}$ is the magnetic permeability of a vacuum. The maximum of $\mu_{0} \partial J_{\varphi} / \partial t$ is normalized to the unit value. The following equations are used in the present paper.

$$
\begin{aligned}
T(t) & =\frac{t}{T_{0}} e^{1-t / T_{0}}, \\
S_{\varphi}(\boldsymbol{r}) & =-e^{-\left(\mu-\mu_{\text {cent }}\right)^{2} / \Delta \mu^{2}} e^{-\left(\nu-v_{\text {cent }}\right)^{2} / \Delta v^{2}},
\end{aligned}
$$

where $\mu$ and $\nu$ are the dipole coordinates $\left(\mu=\cos \theta / r^{2}\right.$, $v=\sin ^{2} \theta / r$ where $\theta$ is the colatitude). Since $\hat{\varphi}$ points westward, negative $J$ indicates the eastward current. Equations (1) and (2) indicate that $J_{\varphi}$ is spatially confined at $\mu=\mu_{\text {cent }}$ and $v=v_{\text {cent }}\left(L_{\text {cent }}=1 / \nu_{\text {cent }}\right)$ to extent of $\Delta \mu$ and $\Delta v$. Figure 3 illustrates the spatial and temporal variation of $\left|\mu_{0} \partial J_{\varphi} / \partial t\right|$ with the parameters of $\Delta \mu=0.001$, $\mu_{\text {cent }}=0, \Delta v=0.01, L_{\text {cent }}=10$, and $T_{0}=5 \mathrm{sec}$. (Note that $J_{\varphi}$ and $\partial J_{\varphi} / \partial t$ are negative because the source current flows eastward.) Figure 3(c) also shows the temporal evolution of $\left|J_{\varphi}\right|$, which grows in time to reach a constant value.

\subsection{Modal analysis of the magnetospheric MHD wave equation}

We solve the MHD equation of the electric perturbations $\left(\delta \boldsymbol{E}_{\perp}\right)$;

$$
\begin{aligned}
& \frac{1}{V_{A}^{2}(\boldsymbol{r})} \frac{\partial^{2} \delta \boldsymbol{E}_{\perp}(\boldsymbol{r}, t)}{\partial t^{2}}+\nabla_{\perp} \times \nabla \times \delta \boldsymbol{E}_{\perp}(\boldsymbol{r}, t) \\
& =-\mu_{0} \frac{\partial \boldsymbol{J}(\boldsymbol{r}, t)}{\partial t}
\end{aligned}
$$

by using modal analysis and Finite Element method (FEM). Modal analysis is an alternative method to the numerical Laplace transform analysis employed by Itonaga et al. (1997a). Modal analysis calculates the contribution of each eigenvector to the external signal. Superposing all contributions of the eigenvectors, we can compose the MHD signal. Here, the eigenvector in the magnetosphere is obtained based on FEM because this method yields accurate eigenvectors in strongly nonuniform system such as the Earth's magnetosphere (Fujita and Patel, 1992). Modal analysis is explained in Appendix A. Equation (A.15) in Appendix A indicates that the signal $\left(\boldsymbol{X}(\boldsymbol{r}, t)=\delta \boldsymbol{E}_{\perp}(\boldsymbol{r}, t)\right)$ is composed of eigenvectors $\left(\boldsymbol{z}_{i}(\boldsymbol{r})\right)$ with temporally varying weights $\left(p_{i}(t)\right)$. We obtain $p_{i}(t)$ from Eqs. (1) and (A.14) as

$$
\begin{aligned}
p_{i}(t)=\frac{G_{i}}{\alpha_{i}}\{ & \frac{T_{0}}{\left(1+s_{i} T_{0}\right)^{2}} e^{1+s_{i} t} \\
& \left.-\frac{t\left(1+s_{i} T_{0}\right)+T_{0}}{\left(1+s_{i} T_{0}\right)^{2}} e^{1-t / T_{0}}\right\},
\end{aligned}
$$


(a)

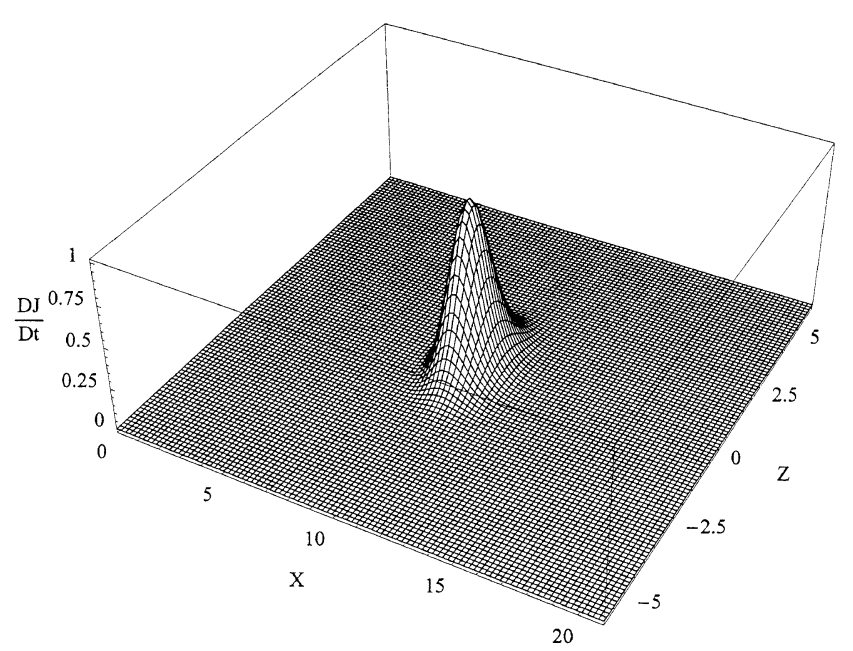

(b)

(c)
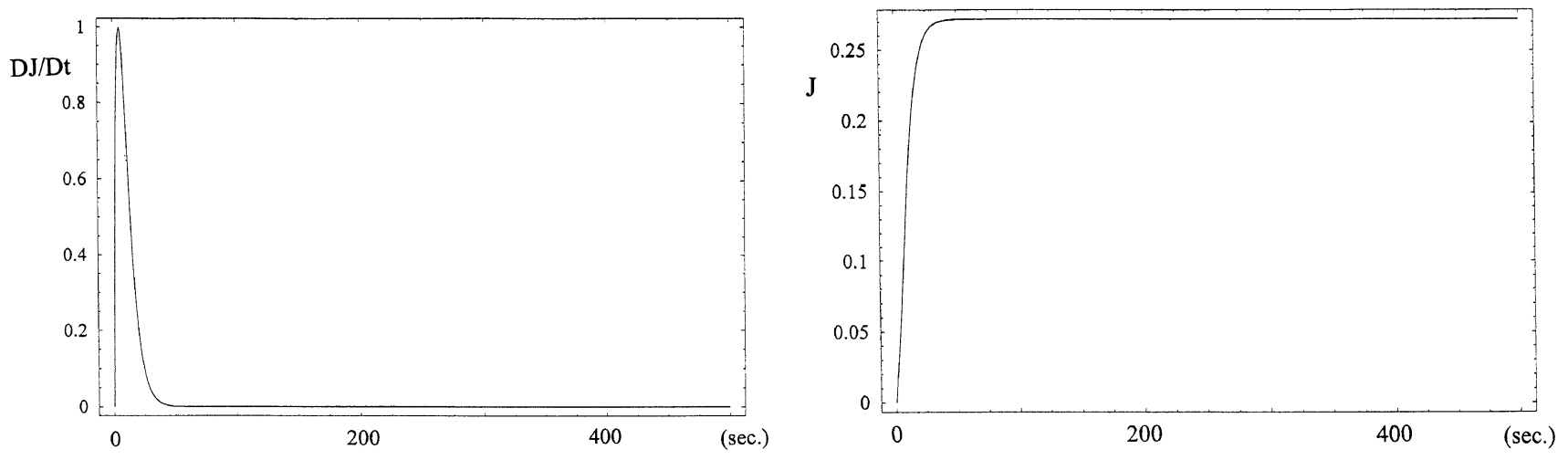

Fig. 3. (a) The spatial profile of $\left|\mu_{0} \partial J_{\varphi} / \partial t\right|$ in $x-z$ coordinates, (b) its temporal variation, and (c) the temporal variation of $\left|\mu_{0} J_{\varphi}\right|$. This current flows eastward. We assign $\Delta \mu=0.001, \Delta v=0.01, L_{\text {cent }}=10$, and $T_{0} \simeq 5 \mathrm{sec}$. The radial and field-aligned extent of the source current is approximately $2 \sim 3 R_{e}$. The maximum of $\left|\mu_{0} \partial J_{\varphi} / \partial t\right|$ is normalized to 1 .

where $G_{i}$ is the $i$-th component of $Z^{T} \boldsymbol{f}(t) / T(t)$ in Eq. (A.13). $G_{i}$ is the product of $Z^{T}$ and the FEM-discretized $\hat{\varphi} S_{\varphi}(\boldsymbol{r}) . s_{i}$ is the eigenfrequency of the $i$-th eigenvector and $\alpha_{i}$ is given in Eq. (A.11). It is clear from Eq. (4) that $p_{i}(t)$ consists of two parts: one is derived from the eigenvector (the first term) and the other is derived from the source current (the second term). Note that only the contribution from the eigenvectors remains at $t \gg T_{0}$.

The temporal accuracy of modal analysis depends on the largest eigenvalue (eigenfrequency). The spatial accuracy is determined by the smallest wavelength of the eigenvectors. No numerical instability appears in modal analysis unlike explicit numerical integral methods such as the two-step Lax Wendroff scheme.

\section{Results}

First, we investigate the general characteristics of the plasmasphere virtual resonance oscillation driven by the source current shown in Fig. 3. Second, we discuss how the source current controls the plasmasphere virtual resonance oscillation.

\subsection{Eigenvector}

The present modal analysis utilizes all electric field eigen- vectors obtained through the numerical calculations in the model that has $44 \times 20$ nodes in the $L \times s$ space $(s$ is the field-aligned direction). The number of eigenvectors used is 817 . The minimum and maximum angular eigenfrequencies employed are $0.00669-0.00137 i \mathrm{sec}^{-1}$ and $83.5 \mathrm{sec}^{-1}$, respectively. Since the source current is symmetric with respect to the equator, only the electric field eigenvectors that are symmetric with respect to the equator have non-zero $G_{i}$.

Before discussing the time-dependent behavior of the MHD signals, let us show the electric field eigenvectors $\left(\delta E_{\varphi}\right)$. Here, the four electric field eigenvectors that have the largest contributions, $G_{i} / \alpha_{i}$ (See Eq. (4)), to the source current are shown in Fig. 4. (The angular eigenfrequencies in $\sec ^{-1}$ are $0.175-0.00235 i, 0.190-0.00210 i, 0.0878-$ $0.00121 i$, and $0.0826-0.00170 i$, from top to bottom.) We notice that all electric field eigenvectors have a larger amplitude in the plasmasphere and expand beyond the plasmapause.

\subsection{MHD signals induced from the source current}

3.2.1 Propagation of the MHD signals in the equatorial plane Since the MHD disturbance is regarded as axisymmetric, it has $\delta E_{\varphi}$ (the azimuthal component of the electric field), $\delta B_{\|}$(the compressional component of the mag- 

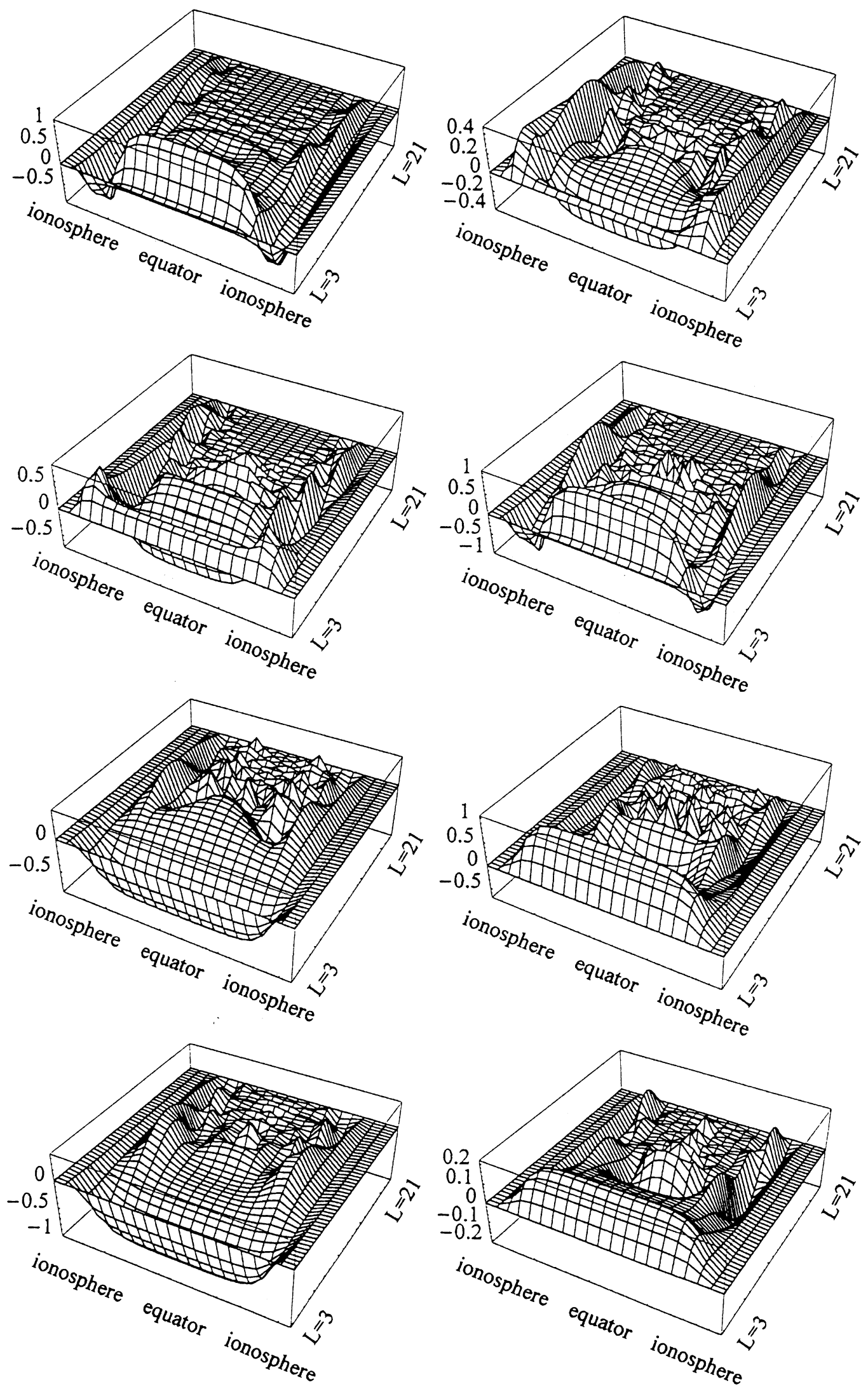

Fig. 4. The left and right panels show the spatial patterns of the real and imaginary parts of the electric field eigenvectors with the four largest $G_{i} / \alpha_{i}$. The figures are shown using the rectangular frame of $\mu \times v$. The angular eigenfrequencies in $\mathrm{sec}^{-1}$ are $0.175-0.00235 i, 0.190-0.00210 i, 0.0878-0.00121 i$, and $0.0826-0.00170 i$ from top to bottom. The electric field eigenvector has an imaginary part due to wave damping associated with an artificial wave absorber in the outer part of the magnetosphere. The amplitude of each eigenvector is normalized to 1. 
(a) $\delta \mathrm{E}_{\Phi}$

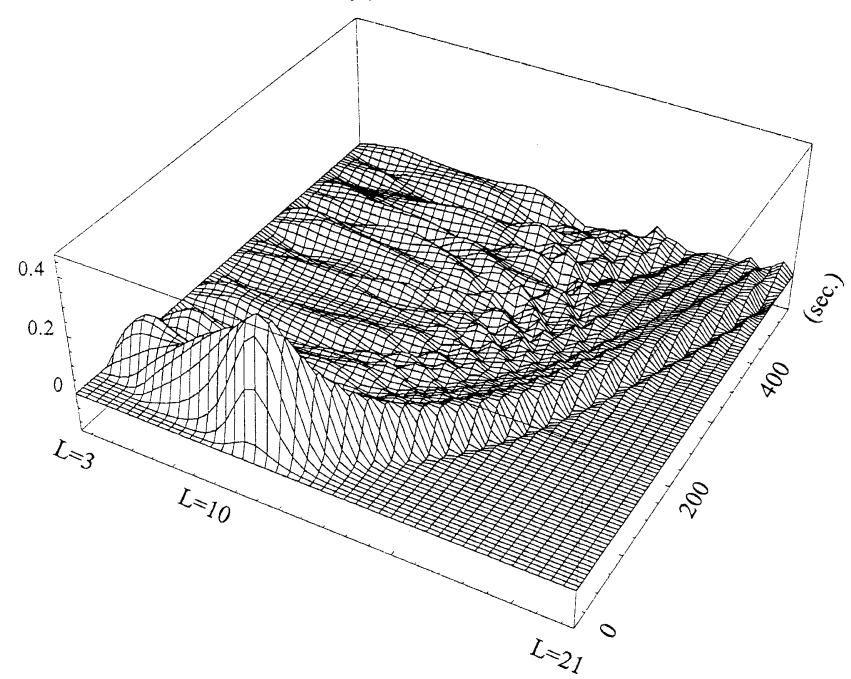

(b) $\delta \mathrm{B}$

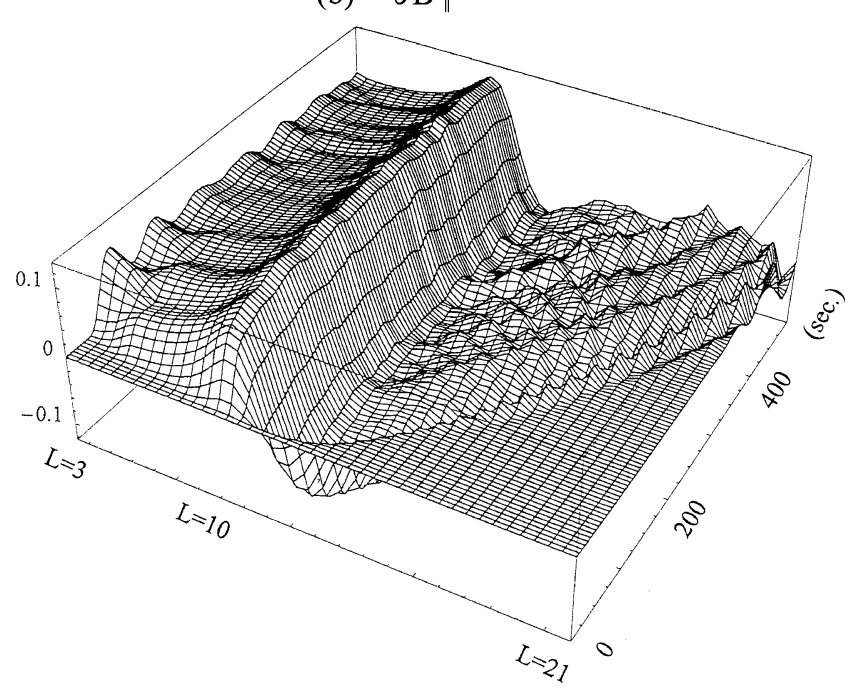

Fig. 5. (a) Temporal variation of $\delta E_{\varphi}$ and (b) that of $\delta B_{\|}$generated by the source current in the equatorial plane. All perturbations are normalized to the maximum intensity of $\left|\mu_{0} \partial J_{\varphi} / \partial t\right|$. It is evident that the perturbations are propagating toward the inner magnetosphere and the outer magnetosphere.

netic field), and $\delta B_{v}$ (the poloidal component of the magnetic field). In the equatorial plane, $\delta B_{v}=0$ when the source current is distributed symmetric with respect to the equator. Therefore, temporal variations in $\delta E_{\varphi}$ and $\delta B_{\|}$in the equatorial plane of $3<L<21$ are illustrated in Fig. 5. Note that the westward $\delta E_{\varphi}$ and the inward $\delta B_{v}$ are positive.

First, we need to check the numerical accuracy of the present results. Physical causality requires that there should be no signal at any position before a wave arrives at that position. On the other hand, in modal analysis, each eigenvector has a contribution at any position and any time (see Eq. (4)). Therefore, reproduction of a propagating wavefront (or cancel-out of contributions from all eigenvectors before arrival of the wavefront) guarantees the accuracy of the present modal analysis calculation. Figure 5 clearly shows that the wavefront in $\delta E_{\varphi}$ and that in $\delta B_{\|}$propagate toward the (a) $\delta \mathrm{E}_{\Phi}$

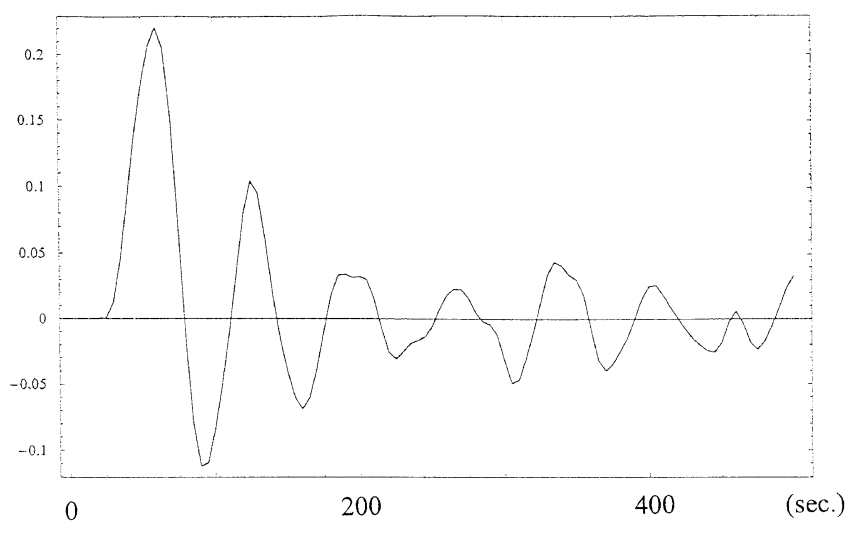

(b) $\delta \mathrm{B}_{\|}$

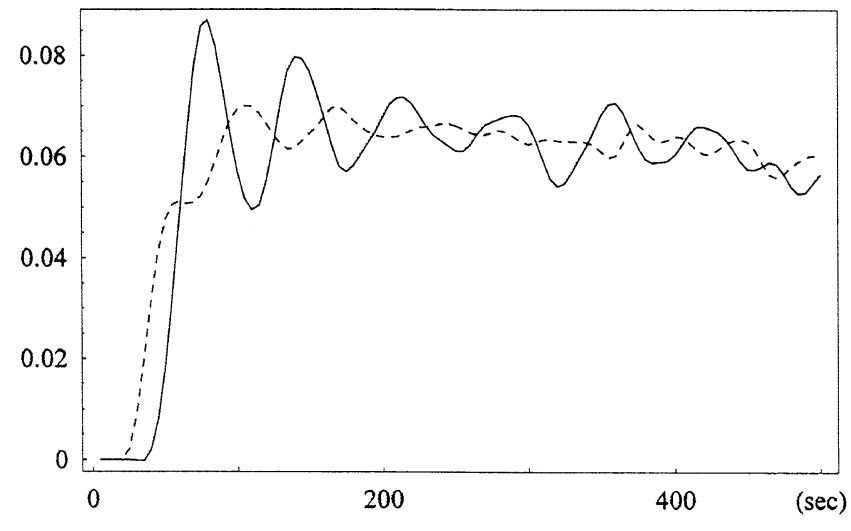

(c) $\mathrm{d} \delta \mathrm{B}_{\|} / \mathrm{dt}$

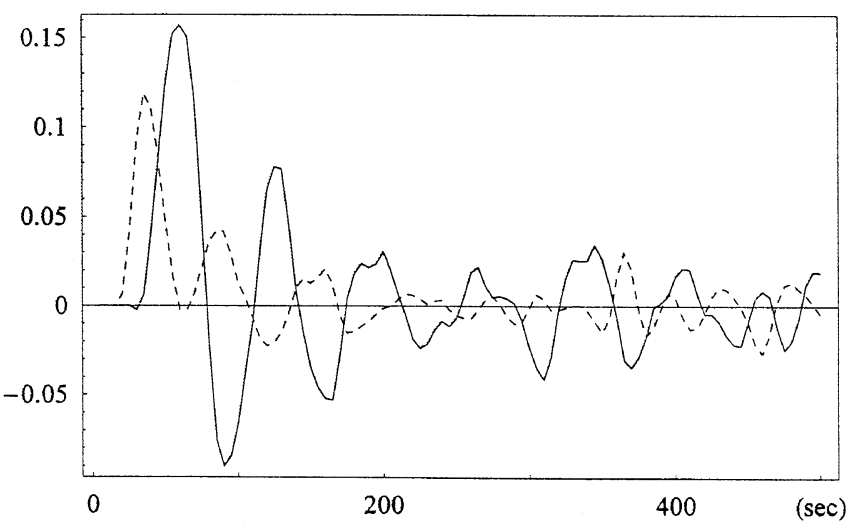

Fig. 6. (a) Waveform of $\delta E_{\varphi}$ at the equator at $L=5.0$, (b) waveform of $\delta B_{\|}$at the equator at $L=3.8$ (full line) and 5.9 (broken line), and (c) waveform of $d \delta B_{\|} / d t$ at the equator at $L=3.8$ (full line) and 5.9 (broken line). It is evident that, after passing the initial disturbance, there remains clear oscillation with a period of approximately $60 \mathrm{sec}$. in $\delta E_{\varphi}$. It is also evident that $\delta B_{\|}$and $d \delta B_{\|} / d t$ have waveforms that change the phase in the inner and outer part of the plasmasphere. This is evidence of the plasmasphere virtual resonance oscillation. Note that westward $\delta E_{\varphi}$ is positive.

plasmasphere and the outer magnetospheres from the center region of the source current $(L=10)$. Therefore, the clear wavefront indicates that the present numerical technique invokes sufficiently accurate results. 
(a) $\delta \mathrm{E}_{\Phi}$

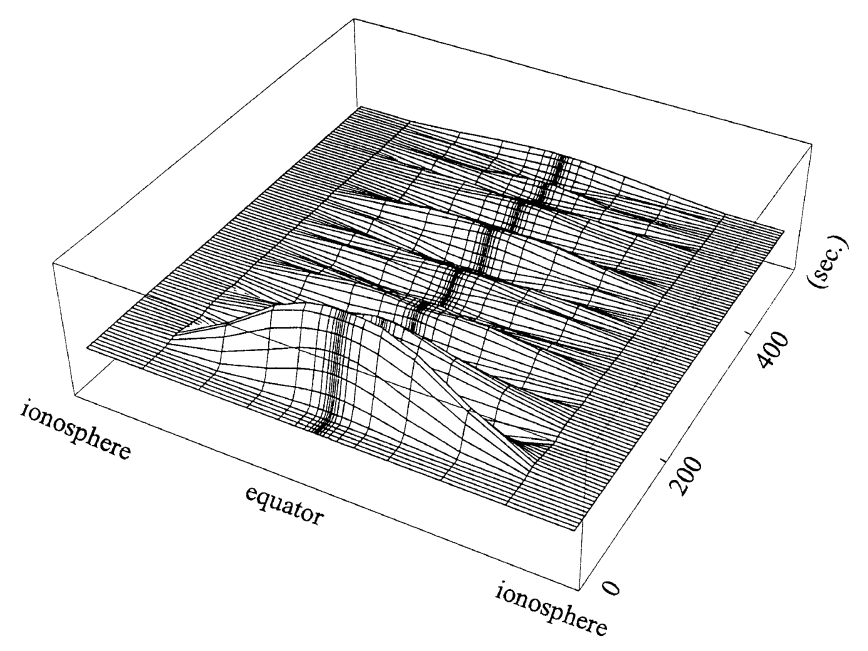

(b) $\mathrm{d} \delta \mathrm{B}_{\|} / \mathrm{dt}$

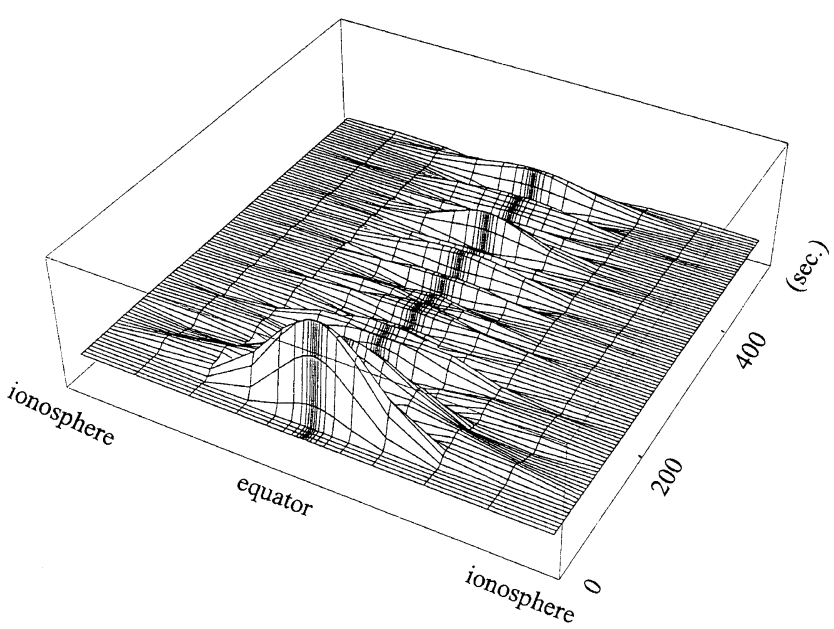

(c) $\mathrm{d} \delta \mathrm{B}_{\nu} / \mathrm{dt}$

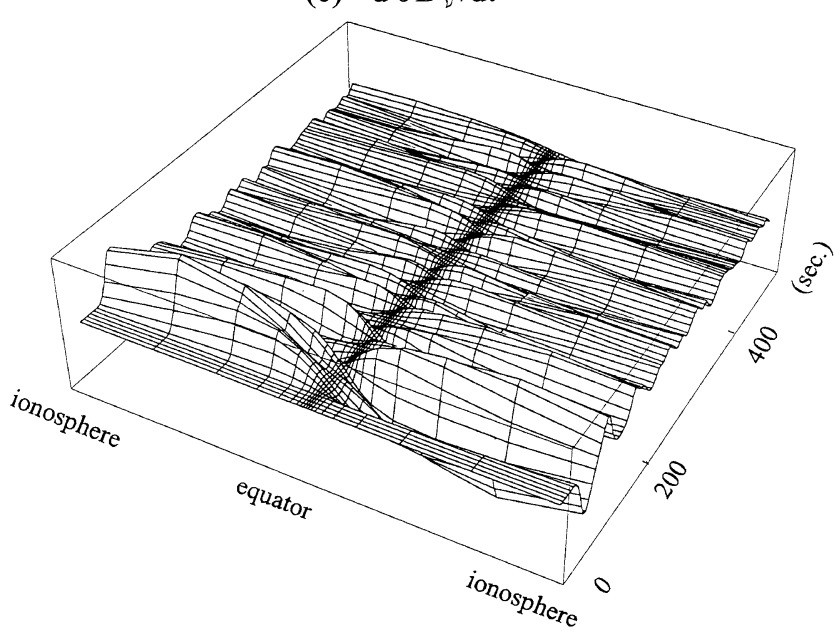

Fig. 7. (a) Temporal evolution of the field-aligned profile of $\delta E_{\varphi}$, (b) that of $d \delta B_{\|} / d t$, and (c) that of $d \delta B_{\nu} / d t$ at $L=5.0$. The horizontal axis is measured using $\lambda(=\arctan (z / x))$. The ionosphere is located at $\pm 60^{\circ} . \delta E_{\varphi}$ and $\delta B_{\|}$are localized in the vicinity of the equator. $\delta B_{\|}$tends to be more confined near the equator compared with $\delta E_{\varphi}$. Note that westward $\delta E_{\varphi}$ and inward $\delta B_{v}$ are positive.

Next, let us describe characteristic features of the signals. $\delta B_{\|}$is null at the center of the source current $(L=10)$. It is positive (northward) in the earthward region and negative (southward) in the opposite region. Bearing in mind that positive $\delta E_{\varphi}$ is westward, the Poynting flux deduced from $\delta E_{\varphi}$ and $\delta B_{\|}$in Fig. 5 is earthward in $L<10$ and tailward in $L>10$. Both $\delta E_{\varphi}$ and $\delta B_{\|}$show rather smoother temporal variation in the inner part of the magnetosphere. This is the plasmasphere virtual resonance oscillation. This will be discussed in detail later. Whereas, ripples in the outer part of the magnetosphere are found to have a period as large as that of the time scale of the source current. (We confirm that these ripples become smaller when the source current has longer time scale variation.) We confirm that the behavior of $\delta E_{\varphi}$ and that of $\delta B_{\|}$in the inner part of the magnetosphere are independent of the artificial wave absorber assigned in the outer part of the magnetosphere. The ripples in the outer magnetosphere are damped due to the wave absorber. In the present paper, we do not consider the behavior of the MHD signal in the outer part of the magnetosphere. The wavefront marching toward the outer boundary arrives at the boundary at the termination time of the simulation. Thus, the outer boundary does not affect the behavior of the MHD signal in the inner magnetosphere during the time interval shown in Fig. 5.

Several theoretical and observational studies have been conducted on plasmasphere virtual resonance oscillation (e.g., Itonaga et al., 1997a,b; Lee, 1998; Takahashi et al., 1992). Let us confirm that the plasmasphere virtual resonance oscillation appears in the present numerical calculation. $\delta E_{\varphi}$ shown in Fig. 5(a) indicates that the oscillation in the plasmasphere extends beyond the plasmapause toward the outer magnetosphere. Namely, $\delta E_{\varphi}$ does not vanish at the plasmapause. Therefore, this oscillation is not the plasmasphere bounded resonance, but may be the plasmasphere virtual resonance oscillation presented by Lee and Kim (1999). $\delta B_{\|}$in Fig. 5(b) has a feature that is typical of the plasmasphere virtual resonance oscillation; the phase relation between $\delta B_{\|}$in the inner plasmasphere and that in the outer plasmasphere is out-of-phase (Takahashi et al., 1992). This 
(a)

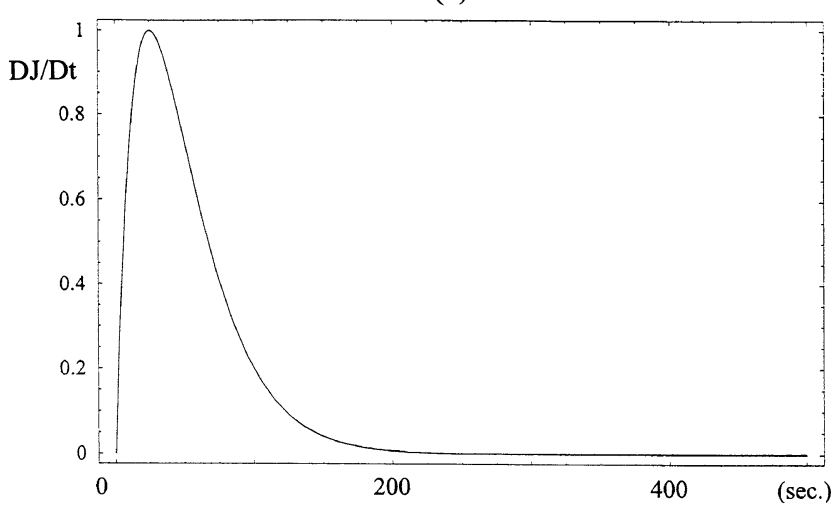

(b)

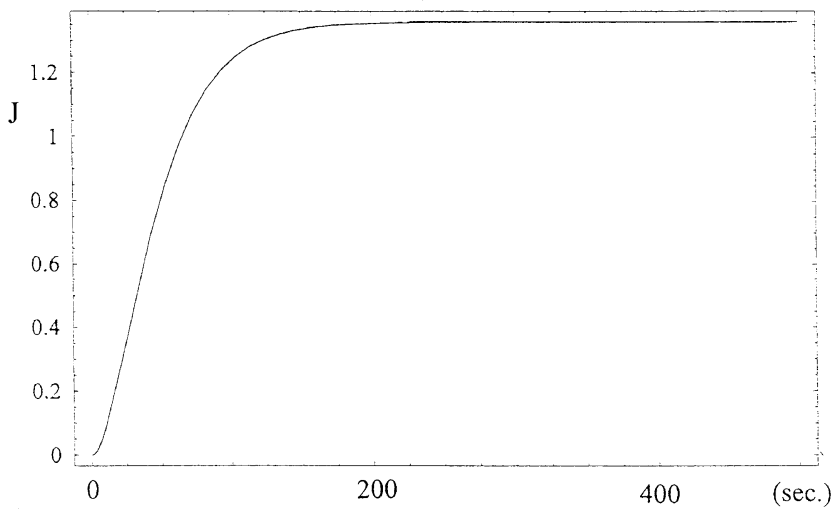

Fig. 8. (a) Temporal variation of $\left|\mu_{0} \partial J_{\varphi} / \partial t\right|$ and (b) that of $\left|\mu_{0} J_{\varphi}\right|$ for $T_{0}=25$ sec. $\Delta \mu=0.001, \Delta v=0.01, L_{\text {cent }}=10$ (same as that in Fig. 3). The maximum of $\left|\mu_{0} \partial J_{\varphi} / \partial t\right|$ is normalized to 1 . This current flows eastward.

indicates that $\delta B_{\|}$is a good indicator of the plasmasphere virtual resonance oscillation. In order to show clearly the waveform of $\delta B_{\|}$in the two regions of the plasmasphere, we present temporal variations of $\delta B_{\|}$at the equator in the inner plasmasphere $(L=3.8)$ and in the outer plasmasphere ( $L=5.9$ ) in Fig. 6. From this figure, $\delta B_{\|}$in the inner plasmasphere and that in the outside of the plasmasphere tend to be out-of-phase. This result confirms that the plasmasphere virtual resonance oscillation exists in the present numerical calculation. Note that the disturbance at $L=3.8$ commenced later than that at $L=5.9$. This is due to the time difference invoked by propagation of the MHD signal in the magnetosphere from $L=5.9$ to 3.8. Since $\delta B_{\|}$has a DC bias due to the growth of the source current, we also show $d \delta B_{\|} / d t$ in Fig. 6(c). This figure also demonstrates the plasmasphere virtual resonance oscillation in the $d \delta B_{\|} / d t$ trace. Finally, the oscillation in $\delta E_{\varphi}$ (Fig. 6(a) is found to be the plasmasphere virtual resonance oscillation because its period is as large as that of $\delta B_{\|}$shown in Fig. 6(b). These results are consistent with those of a previous study by Itonaga et al. (1997a,b), Lee (1998), and Lee and Kim (1999).

3.2.2 Field-aligned profile of the MHD signals Let us examine the field-aligned profiles of the MHD signals in the plasmasphere. The nonuniform $V_{A}$ distribution along the field line influences strongly the amplitude profile of the fast mode oscillation, as explained by Southwood and Kivelson

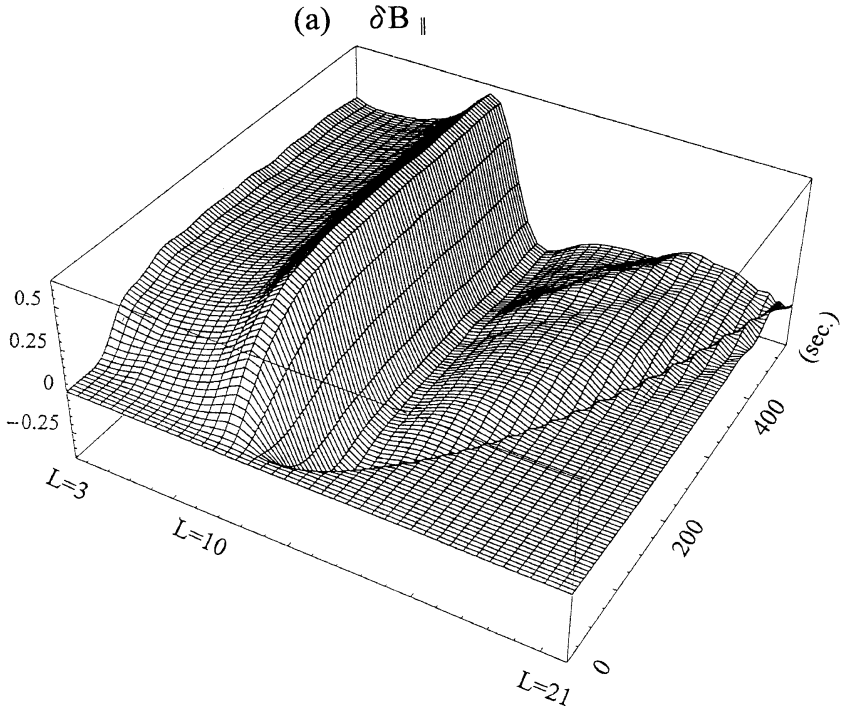

(b) $\delta \mathrm{B}_{\|}$

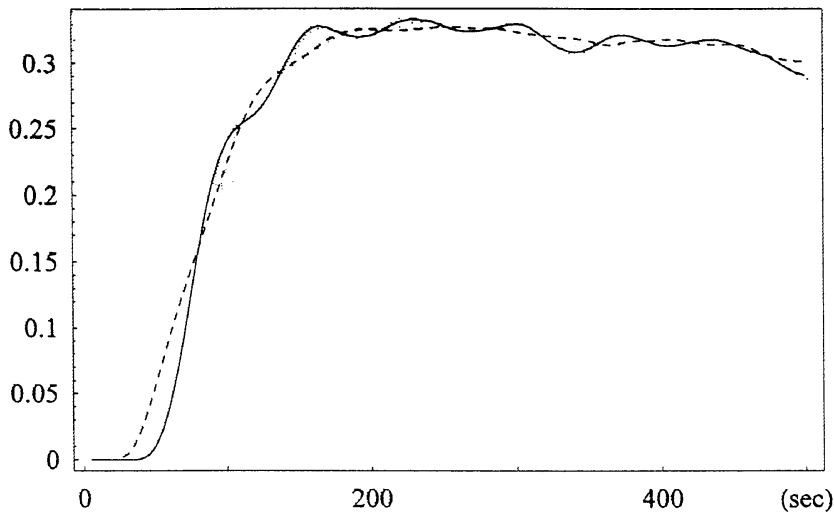

(c) $\mathrm{d} \delta \mathrm{B}_{\|} / \mathrm{dt}$

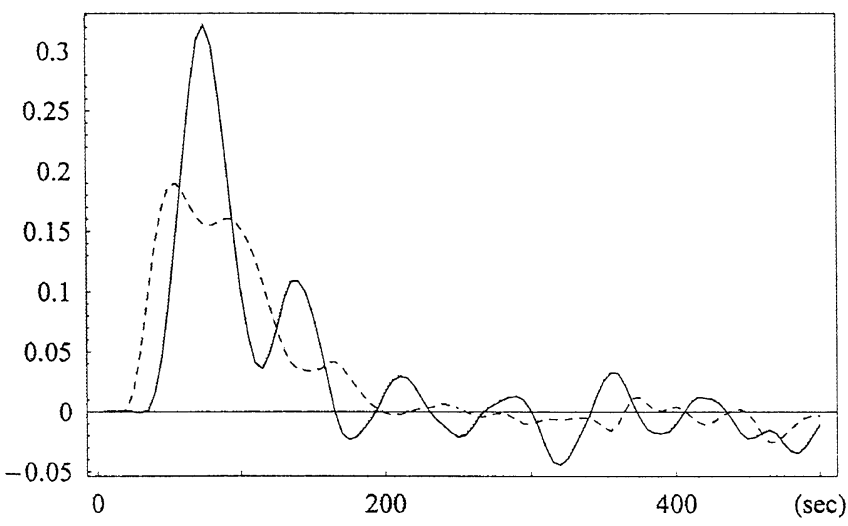

Fig. 9. The waveforms of $\delta B_{\|}$and $d \delta B_{\|} / d t$ at the equator at $L=3.8$ and $L=5.9$ are also shown in (a) and (b). $\Delta \mu=0.001, \Delta v=0.01$, $L_{\text {cent }}=10$ (the source shown in Fig. 3).

(1986). Lee (1996) also demonstrated that the fast mode wave does not reach the ionosphere by calculating the cutoff frequency of the fast mode wave. However, no report has shown the field-aligned profile of the electromagnetic field perturbations associated with the plasmasphere virtual resonance oscillation in the dipole magnetosphere. We calculate 
(a)

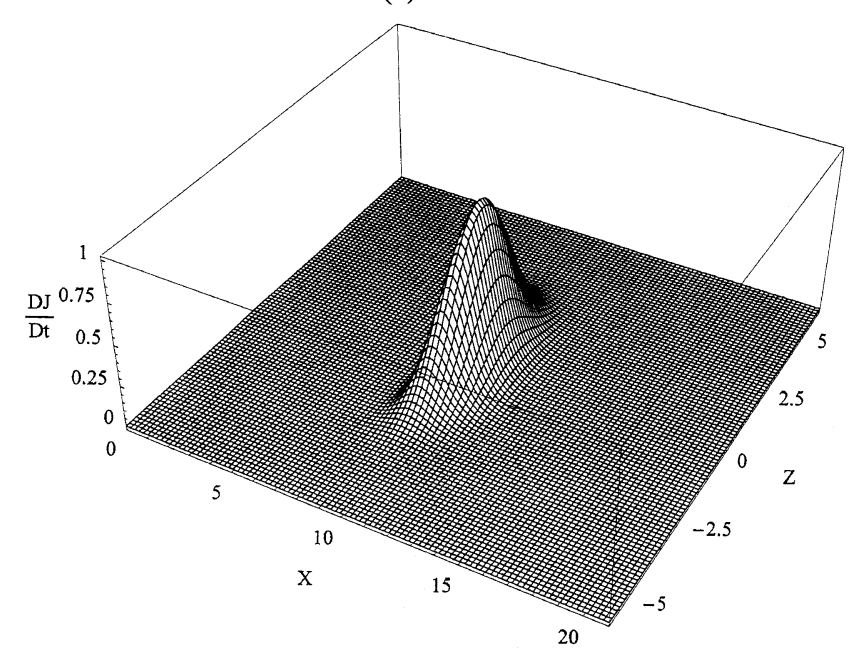

(b)

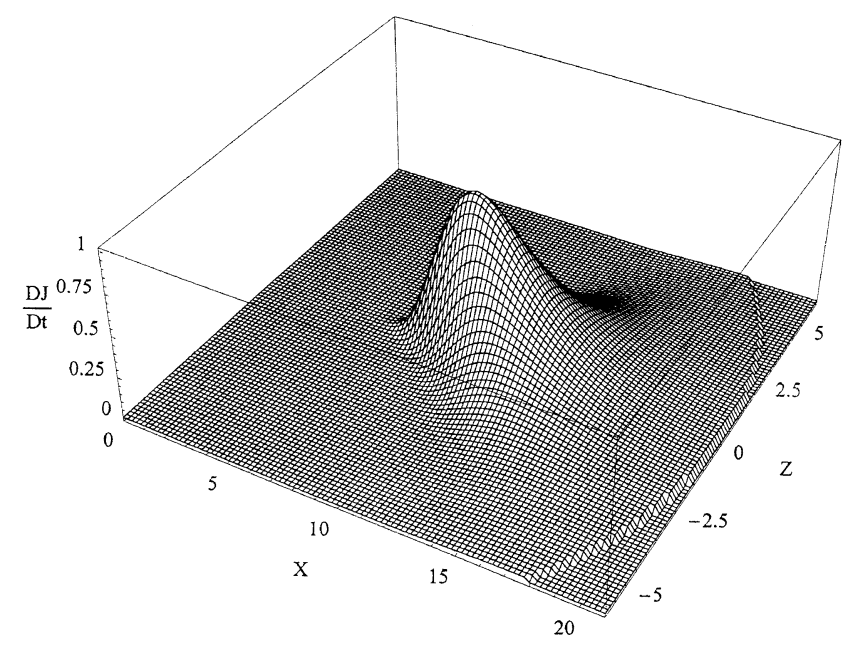

(c)

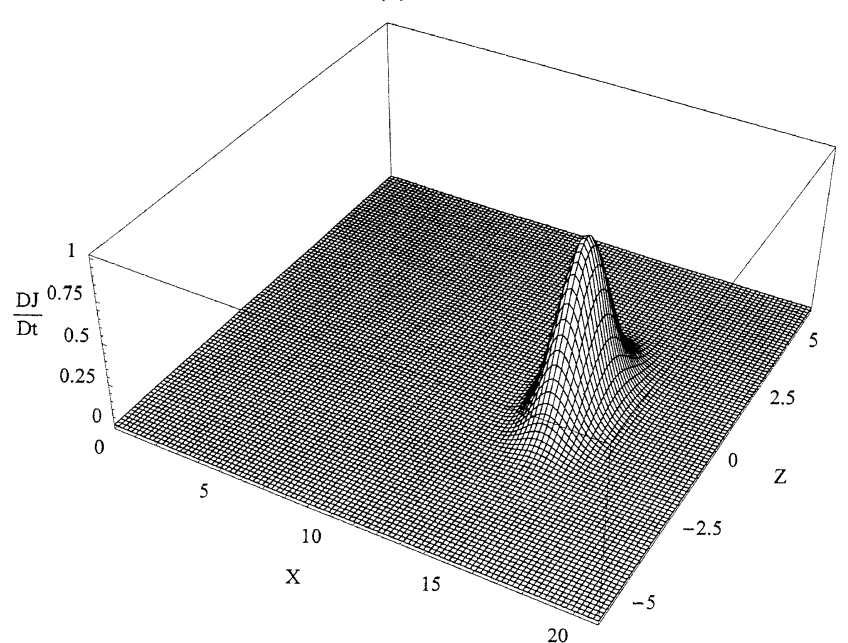

Fig. 10. Spatial profiles of the source current: (a) extended in the field-aligned direction $\left(\Delta \mu=0.002, \Delta v=0.01, L_{\text {cent }}=10\right)$; (b) extended in the radial direction $\left(\Delta \mu=0.001, \Delta v=0.03, L_{\text {cent }}=10\right)$; and (c) located in the outer magnetosphere $\left(\Delta \mu=0.0006, \Delta v=0.008, L_{\text {cent }}=15\right)$.

the field-aligned profile of the perturbations, which are comparable to the observations by AMPTE CCE (Takahashi et al., 1995) and by AKEBONO (Osaki et al., 1998).

Figure 7 illustrates temporal variations in the field-aligned profiles of $\delta E_{\varphi}$ in (a), $d \delta B_{\|} / d t$ in (b), and $d \delta B_{v} / d t$ in (c). (In order to pick up only the oscillatory variation, we show $d \delta B_{\|} / d t$ and $d \delta B_{v} / d t$ instead of $\delta B_{\|}$and $\delta B_{\nu}$.) Signals at $L=5.0$ (where $V_{A}$ is minimized) are employed. The "ionosphere" is located at the latitude $(\lambda)$ of $60^{\circ}$ for $\delta E_{\varphi}$ in (a) and $\delta B_{\|}$in (b), where $\lambda$ is defined as $\arctan (z / x)$. (See Fig. 2 for definitions of $z$ and $x$.) It is evident that $\delta E_{\varphi}$ and $d \delta B_{\|} / d t$ are confined near the equator. In particular, $\delta B_{\|}$is almost confined in the region of $|\lambda|<20^{\circ} \sim 30^{\circ}$. (Although not shown here, we confirm that this feature is independent of the field-aligned profile of the source current as long as the field-aligned width of the source current is not larger than $4 R_{e}$, twice as large as that shown in Fig. 3(a)). It is noteworthy that the equatorial confinement of $\delta B_{\|}$depends on the field-aligned profile of $V_{A}$. Moreover, close observation of Figs. 7(a) and (b) reveals that $d \delta B_{\|} / d t$ tends to be con- fined in the vicinity of the equator compared with $\delta E_{\varphi}$. The equatorially localized compressional perturbation is consistent with the observational results obtained by Takahashi $e t$ al. (1995) and Osaki et al. (1998). In particular, Osaki et al. (1998) revealed that the compressional magnetic perturbation of the Pi2 signal in the magnetosphere is not observed by AKEBONO at $\lambda_{m}>20^{\circ}$.

Next, $d \delta B_{v} / d t$ in Fig. 7(c) is investigated. $\delta B_{v}$ is calculated based on $\delta E_{\varphi}$ in the present calculation. Since the central difference scheme used in the calculation of $\delta B_{\nu}$ shifts the lowermost point of the field line from $60^{\circ}$ (for $\delta E_{\varphi}$ and $\left.\delta B_{\|}\right)$to $45^{\circ}$. Since the source signal is given as symmetric with respect to the equator, $\delta B_{v}$ is always diminished at the equator. Thus, $\delta B_{v}$ shows the field-aligned odd-mode structure. In addition, $\delta B_{v}$ can has a relatively larger signal intensity near the ionosphere.

\subsection{Plasmasphere virtual resonance oscillation and spa- tial and temporal variations of the source current}

We have investigated in detail the behavior of the plasmasphere virtual resonance oscillation induced by the source 
(a) $\delta \mathrm{B}_{\|}$

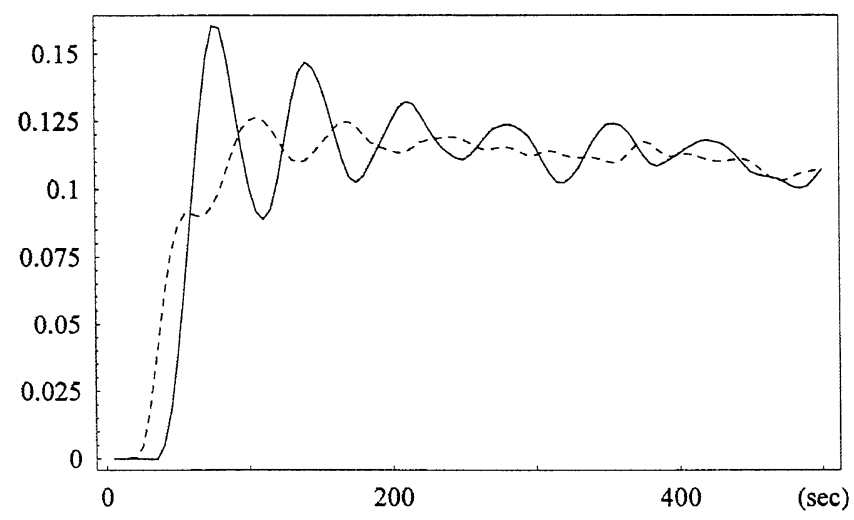

(b) $\mathrm{d} \delta \mathrm{B}_{\|} / \mathrm{dt}$

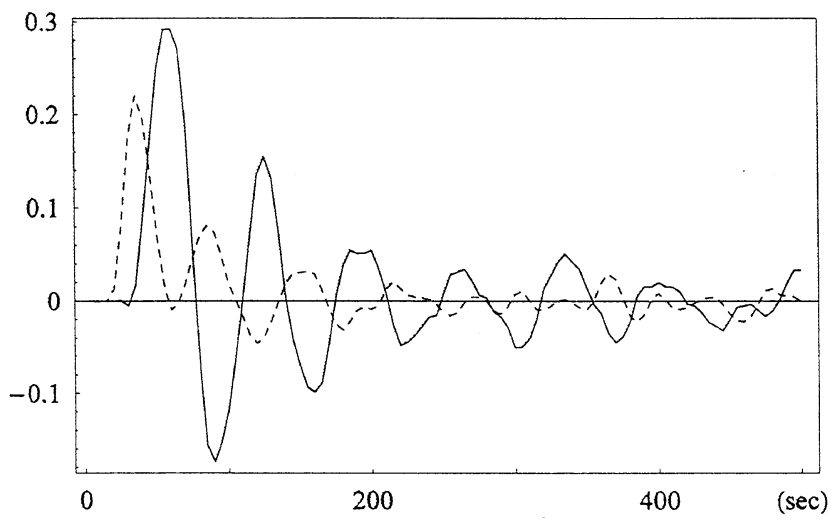

Fig. 11. (a) Temporal evolution of $\delta B_{\|}$and (b) that of $d \delta B_{\|} / d t$ in the equatorial plane. The full and broken lines indicate the waveforms at $L=3.8$ and at $L=5.9$, respectively. The source current is assigned with $\Delta \mu=0.002, \Delta v=0.01, L_{\text {cent }}=10$ (the source shown in Fig. 11(a)). $T_{0}=5 \mathrm{sec}$.

current shown in Fig. 3. Here, let us examine how the plasmasphere virtual resonance oscillation is controlled by the temporal and spatial variations of the source current. Since compared with $\delta E_{\varphi}, \delta B_{\|}$clearly shows the plasmasphere virtual resonance oscillation, only $\delta B_{\|}$is investigated here.

3.3.1 Temporal variation of the source current Itonaga et al. (1997b) investigated the MHD response of the inner magnetosphere for rapidly and gradually growing source impulses. They manifested that the rapid impulse invokes a clear sinusoidal damped oscillation in the inner magnetosphere. Here, let us confirm their results in the dipole magnetosphere model. Temporal evolution of $\left|\mu_{0} \partial J_{\varphi} / \partial t\right|$ and that of $\left|\mu_{0} J_{\varphi}\right|$ with $T_{0}=25 \mathrm{sec}$. are shown in Fig. 8. Note that the maximum of $\left|\mu_{0} \partial J_{\varphi} / \partial t\right|$ is normalized to 1 . The spatial variation of the source current is as described earlier in the present study (Fig. 3). By using this source current, we calculate the equatorial variation of $\delta B_{\|}$with a longer time scale $\left(T_{0} \simeq 25 \mathrm{sec}\right.$.) in Fig. 9. Figures $9(\mathrm{a})$ and (b) show the waveforms of $\delta B_{\|}$and $d \delta B_{\|} / d t$ at $L=3.8$ and $L=5.9$. Figures 9(a) and (b) and Figs. 5(b) and (c) show that the plasmasphere virtual resonance oscillation in $\delta B_{\|}$is clearer for $T_{0}=5 \mathrm{sec}$. Note that $d \delta B_{\|} / d t$ shows clear plasmasphere virtual resonance oscillation in both cases. Since (a) $\delta \mathrm{B}_{\|}$

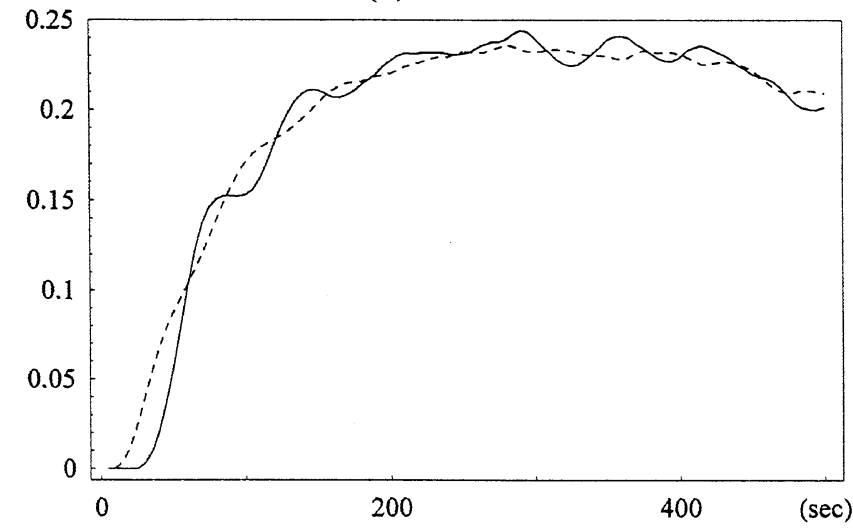

(b) $\mathrm{d} \delta \mathrm{B} \| / \mathrm{dt}$

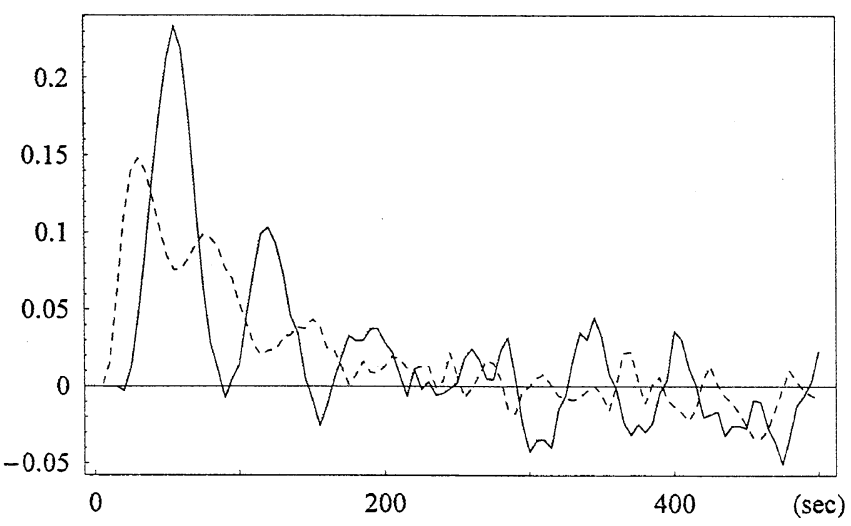

Fig. 12. Same as Fig. 11. The source current is assigned with $\Delta \mu=0.001$, $\Delta v=0.03, L_{\text {cent }}=10$ (the source shown in Fig. 11(b)). $T_{0}=5 \mathrm{sec}$.

the Pi2 observed in the plasmasphere and in the low-latitude ground shows various waveforms, the cases shown in Figs. 5 ( $T_{0}=5 \mathrm{sec}$.) and $10\left(T_{0}=25 \mathrm{sec}\right.$.) may both exist. It is noteworthy that the temporal evolution of the source current controls the waveform.

3.3.2 Spatial distribution of the source current It is interesting to understand how the response of the inner magnetosphere is controlled by the spatial distribution of the source impulse. Figure 10 summarizes the spatial profiles of the source currents employed here. Figures 10(a), (b), and (c) present respectively the source extended in the fieldaligned direction (field-aligned extent $\sim 4 \mathrm{R}_{e} ; \Delta \mu=0.002$, $\left.\Delta v=0.01, L_{\text {cent }}=10\right)$, that extended in the radial direction (radial extent $\sim 4 \mathrm{R}_{e} ; \Delta \mu=0.001, \Delta v=0.03, L_{\text {cent }}=10$ ), and that located in the outer magnetosphere $(\Delta \mu=0.0004$, $\left.\Delta v=0.005, L_{\text {cent }}=15\right)$. Figures 11,12 , and 13 illustrate the waveforms of $\delta B_{\|}$and $d \delta B_{\|} / d t$ in the equatorial plane at $L=3.8$ and $L=5.9$.

Figures 6 and 11 indicate that $\delta B_{\|}$and $d \delta B_{\|} / d t$ behave in a similar manner, with the exception of the amplitude when the field-aligned profile of the source current is extended and the radial extent is the same. Note that the amplitude of the oscillation becomes larger when the field-aligned extent becomes larger. For a source (Fig. 11) twice as large the reference one (Fig. 6), the amplitude doubles. On the 
(a) $\delta \mathrm{B}_{\|}$

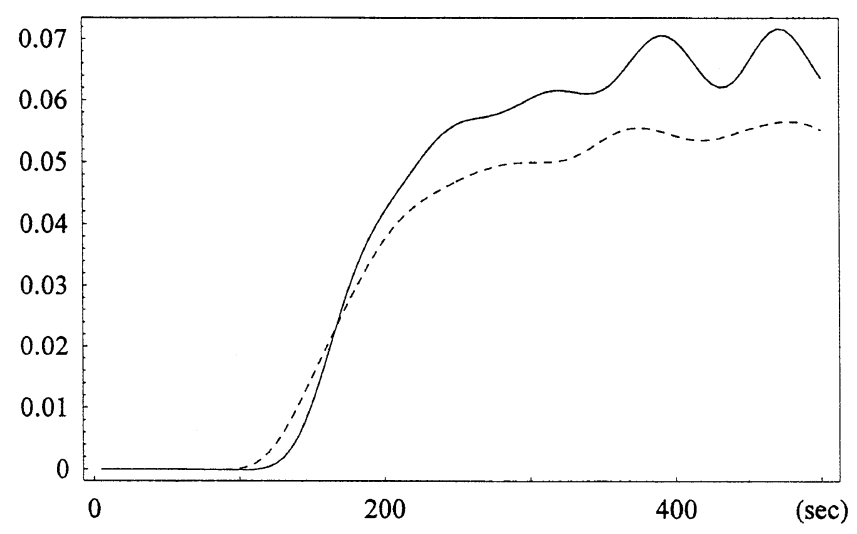

(b) $\mathrm{d} \delta \mathrm{B}_{\|} / \mathrm{dt}$

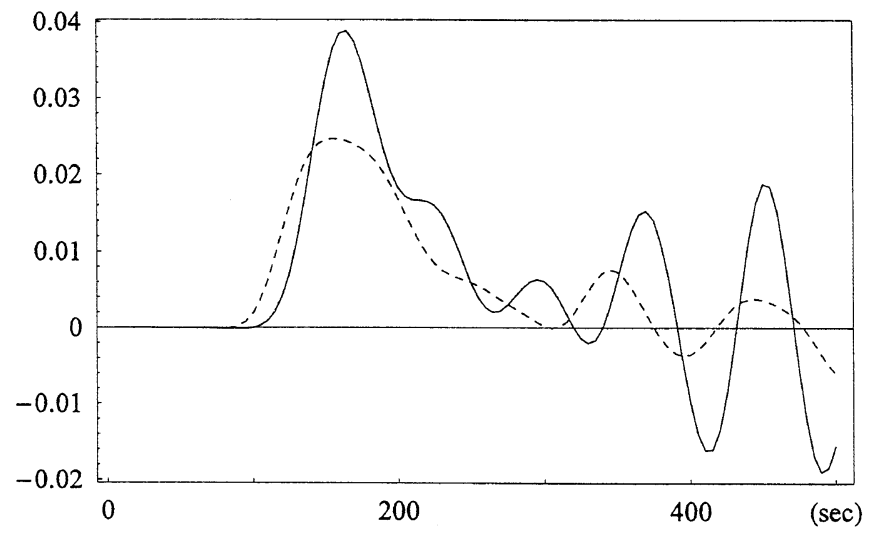

Fig. 13. Same as Fig. 11. The source current is assigned with $\Delta \mu=0.0004$, $\Delta v=0.005, L_{\text {cent }}=15$ (the source shown in Fig. 11(c)). $T_{0}=5 \mathrm{sec}$.

other hand, when the source current is extended in the radial direction, the plasmasphere virtual resonance oscillation in the $\delta B_{\|}$trace becomes rather obscure (Fig. 12). The $\delta B_{\|}$ waveform becomes similar to that for $T_{0}=25 \mathrm{sec}$. (Fig. 8). Moreover, $d \delta B_{\|} / d t$ contains shorter-period waves compared to that for $T_{0}=25 \mathrm{sec}$. The signal detected in the plasmasphere is composed of the components emitted from all places of the spatial extent of the source current. When the source current is extended in the radial direction, the signal emitted in the nearest part and that in the farthest part of the source current region have a significant phase difference. By summing up all of the signals emitted from all parts of the source current, the plasmasphere virtual resonance oscillation becomes obscure. This is the reason that the plasmasphere virtual resonance oscillation becomes slightly unclear when the source current is extended in the radial direction.

Next, let us investigate the dependence of the plasmasphere virtual resonance oscillation on the location of the source current (Fig. 13). This figure shows $\delta B_{\|}$and $d \delta B_{\|} / d t$ at $L=3.8$ and $L=5.9$ for the source current with $L_{\text {cent }}=$ 15. (In order to avoid the effects of the artificial waveabsorbing layer and the outer boundary, we change the model in which the outer boundary is located at $L_{\text {out }}=35$ and the wave-absorbing layer is placed at $L>30$.) Comparing the signal behavior for $L_{\text {cent }}=10$ (Fig. 6) and that of $L_{\text {cent }}=15$

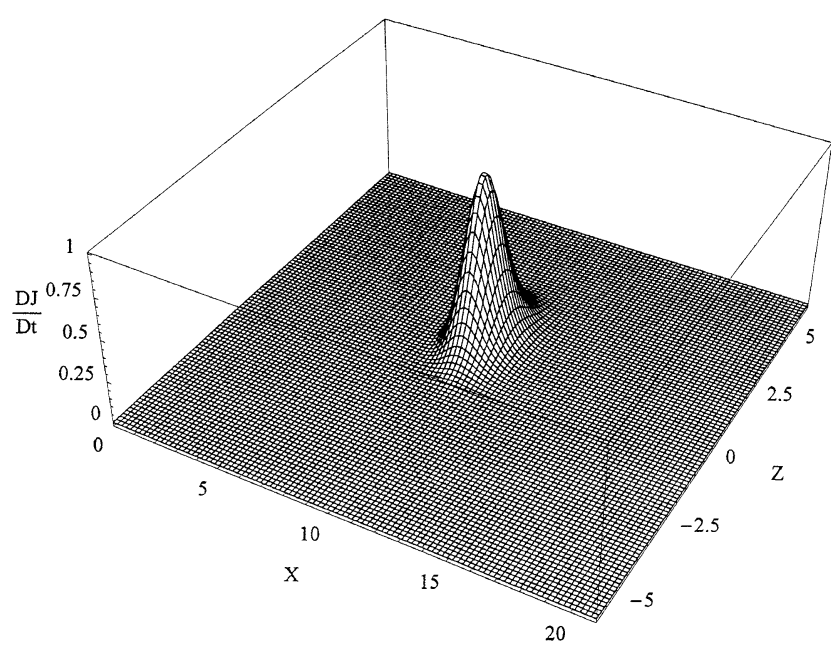

Fig. 14. Spatial profiles of the source current located at about $1 R_{e}$ apart from the equator $\left(\mu_{\text {cent }}=0.001, \Delta \mu=0.001, \Delta v=0.01, L_{\text {cent }}=10\right)$.

(a) $\mathrm{L}=3.8$

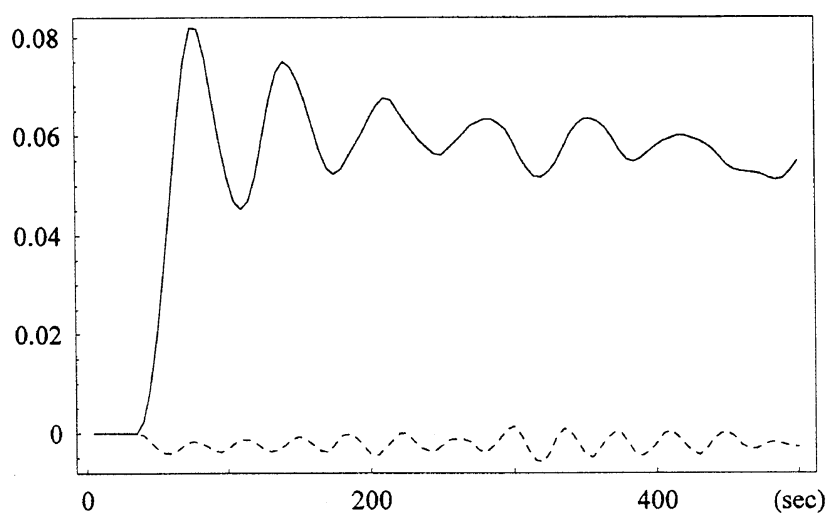

(b) $\mathrm{L}=5.9$

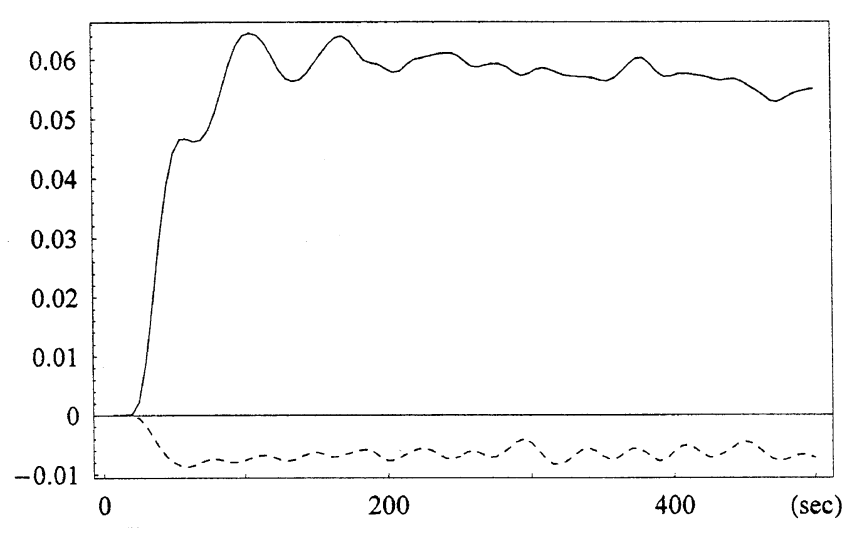

Fig. 15. Waveforms of $\delta B_{\|}$(full line) and $\delta B_{v}$ (broken line) in the equatorial plane for the source current shown in Fig. 15. (a) waveforms at $L=3.8$ and (b) at $L=5.9 . T_{0}=5 \mathrm{sec}$.

(Fig. 13), the latter has the plasmasphere virtual resonance oscillation appearing considerably after the commencement of the initial signal. This feature is quite different from the typical Pi2 waveform. Namely, this may indicate that Pi2 
(a) $\mathrm{L}=3.8$

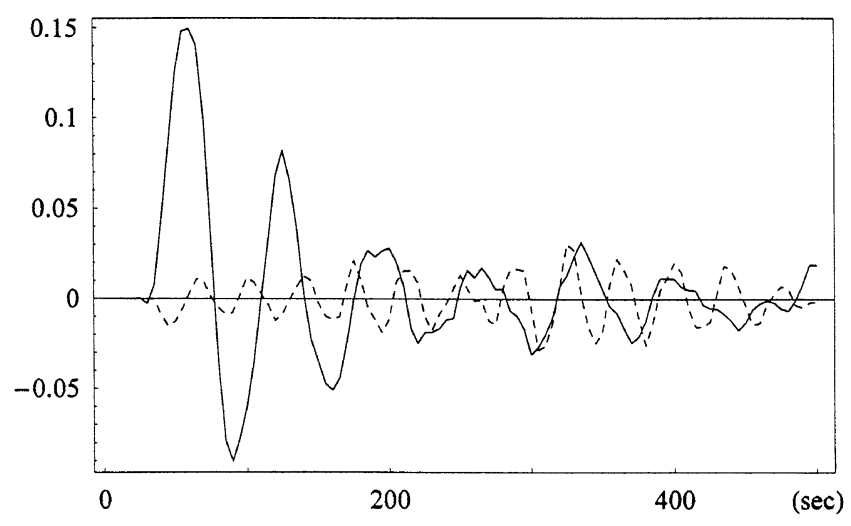

(b) $\mathrm{L}=5.9$

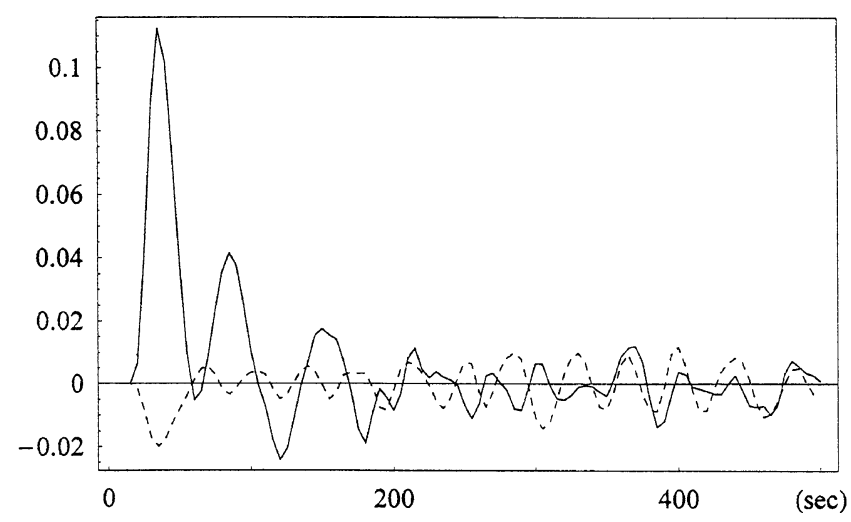

Fig. 16. Waveforms of $d \delta B_{\|} / d t$ (full line) and $d \delta B_{v} / d t$ (broken line) in the equatorial plane for the source current shown in Fig. 15. (a) waveforms at $L=3.8$ and (b) at $L=5.9$. $T_{0}=5 \mathrm{sec}$.

pulsation is generated when the source current is located within $L=10$.

\subsection{Source current located in the off-equator region}

So far, we have investigated MHD signals generated by the source current for which the spatial distribution is symmetric with respect to the equator. Here, let us consider the case in which the source current is asymmetric. This is a likely case in SSC events, in which the solar wind shock impinging on the magnetopause has a shock front that is not vertical to the equatorial plane of the Earth. (However, since the source current for $\mathrm{Pi} 2$ seems to be symmetric with respect to the equator, the off-equatorial source current may be an unlikely case in the Pi2 event.) In order to investigate the effect of this asymmetry, we employ the source current located in the off-equator region. Thus, $\mu_{\text {cent }}$ in Eq. (2) is set as 0.001, which corresponds to the center of the source current located approximately $1 R_{e}$ from the equator, as shown in Fig. 14 .

Figures 15 and 16 illustrate the waveforms of $\delta B_{\|}$and $\delta B_{v}$ in the equatorial plane at $L=3.8$ and $L=5.9$. $\delta B_{v}$ has non-zero amplitude at the equator in the case of the offequatorial source current. This is in contrast to the case of the equatorial source current. In other words, when the poloidal component $\left(\delta B_{v}\right)$ of the MHD signal is detected by satellite at the equator, we may guess that the source current is asymmetric with respect to the equator. Next, from
Figs. 6,15 , and 16 , we find that $\delta B_{\|}$does not change greatly when the source current is located in the off-equatorial region. $\delta B_{v}$ has a shorter frequency than $\delta B_{\|}$. This feature is also evident in $d \delta B_{\nu} / d t$ and $d \delta B_{\|} / d t$. Here, we refer to the symmetric mode/anti-symmetric mode as the eigenvector having the electric field symmetric/anti-symmetric with respect to the equator. The symmetric mode has zero $\delta B_{v}$, non-zero $\delta B_{\|}$, and non-zero $\delta E_{\varphi}$ at the equator. On the other hand, the anti-symmetric mode has non-zero $\delta B_{v}$, zero $\delta B_{\|}$, and zero $\delta E_{\varphi}$ at the equator. In the case of the equatorial source current, only the symmetric modes are generated. In the case of the off-equatorial source current, both symmetric and anti-symmetric modes are generated. Therefore, for the off-meridional source current, at the equator, $\delta B_{\|}$consists of only the symmetric modes and $\delta B_{v}$ consists of only the anti-symmetric modes. Bearing in mind that the fundamental symmetric mode has a longer eigenperiod than the anti-symmetric mode with the longest eigenperiod, we understand that $\delta B_{\|}$has a longer period than $\delta B_{v}$. Note that the period of $\delta B_{\|}$is not simply twice as large as that of $\delta B_{v}$. In other words, the waveform of $\delta B_{v}$ seems to be independent of that of $\delta B_{\|}$. One of the reasons for this is that the ratio of the eigenperiods of the fundamental and second harmonic eigenvectors is not 1:2 in nonuniform systems such as the Earth's magnetosphere. Another reason is that several eigenvectors contribute to $\delta B_{\|}$and $\delta B_{v}$ at the same time. Therefore, $\delta B_{\|}$ and $\delta B_{v}$ may be detected as independent oscillations when the source current is asymmetric with respect to the equator.

\section{Discussion and Conclusions \\ 4.1 Discussion}

We have determined that the plasmasphere virtual resonance oscillation of the compressional magnetic perturbation $\left(\delta B_{\|}\right)$has a waveform that depends on the temporal variation of the source current and the radial extent of the source current. The plasmasphere virtual resonance oscillation has a clear oscillatory waveform with the period of typical Pi2 pulsation when the source current is confined in the radial direction to the extent of approximately $1 \sim 2 R_{e}$. When the source current spreads wider in the $L$ direction, the waveform in the plasmasphere becomes unclear. At the same time, the induction observation $\left(d \delta B_{\|} / d t\right)$ detects the shorter frequency component. On the other hand, as the rise time of the source current becomes longer, the waveform becomes similar to the case of the wider source current. However, the induction record does not have the shorter-period component. These results indicate the possibility for evaluating the spatial and temporal behavior of the source current from the observation of the Pi2 pulsation in the plasmasphere when the $V_{A}$ model in the magnetosphere is given.

Next, the present model suggests that the source current should be located at $L \leq 10$ for the plasmasphere virtual resonance oscillation with typical Pi2 waveform. Note that the magnetosphere model depicted in Fig. 2 represents a rather quiet magnetosphere. Usually, the plasmapause is located at approximately $L=4 \sim 5$. In this case, the location of the source current may be closer to the Earth. The "depth" of $V_{A}$ in the plasmasphere affects the waveform of the plasmasphere resonance oscillation. In any condition of the magnetospheric activity, the location of the source current that 
produces the typical $\mathrm{Pi} 2$ pulsation in the plasmasphere may not shift far away from the plasmapause. This location of the source current is consistent with the location of the current disruption (Takahashi et al., 1987; Lui, 1996). Note that the present numerical analysis uses the dipole magnetosphere. This model is valid in $L<10$. The waveform of the compressional perturbation based on the source current located at $L=15$ in our numerical calculation may be modified due to the fact that the magnetosphere becomes different from the dipole magnetosphere model. However, the plasmasphere virtual resonance oscillation may not have a waveform that is similar to the typical Pi2 signal because $V_{A}$ can be regarded to decrease in the radial direction, similar to that in the dipole magnetosphere.

The azimuthally uniform source current model is equivalent to the case in which $m$ (the azimuthal wave number) is zero. Thus, the coupled oscillation is not considered in the present paper. As discussed by Fujita and Patel (1992), the compressional magnetic field does not exhibit singular behavior on the field line where field line resonance occurs. Therefore, as long as the compressional component is treated, no field-line resonance occurs. Next, Lee (1996) found that the plasmasphere virtual resonance oscillation depends on $m$. Namely, a source signal having smaller $m$ can penetrate deep into the plasmasphere. Satellite observations in the inner magnetosphere revealed that the Pi2 pulsations appear only in the nightside (Takahashi et al., 1992). This observation suggests that $m$ of the Pi2 pulsation in the plasmasphere should be $2 \sim 3$. Therefore, the amplitude of the plasmasphere virtual resonance oscillation may be reduced compared to the present numerical results. As long as $m$ is not excessively large, the results obtained in the present paper may remain valid in the meridian of the center longitude of the source current (midnight). The $m$-control of the plasmasphere virtual resonance oscillation is our next problem. In addition, in the case of nonzero- $m$, the fast mode wave couples to the Alfvén wave and the field-aligned currents carried by the Alfvén waves are dissipated at the ionosphere by conductivity. This leads to damping of the pulsation and Poynting flux that is directed toward the ionosphere. Osaki et al. (1998) showed that the Poynting flux associated with the magnetospheric Pi2 pulsation is directed away from the equator. However, the field-aligned Poynting flux vanishes in our simulation because the fast mode wave is perfectly reflected by the ionosphere. Therefore, our simulation could reveal only the plasmasphere virtual resonance oscillation seen in the compressional component associated with the $\mathrm{Pi} 2$ pulsation. Inclusion of a realistic ionospheric conductivity and coupled oscillation is an important subject of future study with respect to the simulation.

In the present paper, the source current itself does not move; rather, only its intensity varies in time. However, this assumption may be simplified. For example, the SSC pushes the magnetopause toward the Earth. Then, the ChapmanFerraro current changes its location and intensity in time. The current disruption region also moves from the nearEarth magnetotail toward the deep magnetotail (Ohtani et al., 1992). In future studies, the moving source current must be taken into consideration. At the same time, a more realistic magnetosphere model that describes well the magnetotail in the nightside must be used.

The source current model adopted herein is a simplified model. Physically speaking, the current disruption cannot be described in terms of the MHD theory (Lui, 1996) and the cold plasma approximation is not valid in the near-Earth magnetotail region in the substorm period. The source current employed in the present paper is an "equivalent" current that produces the plasmasphere virtual resonance oscillation of the Pi2 pulsation. Direct observation of the plasma and field in the current disruption region indicates that the current system is temporarily and spatially complex compared to the present current system (Takahashi et al., 1987). Not all of the components of such a complex current system are likely to propagate to the plasmasphere to form the plasmasphere virtual resonance oscillation. The current system averaged over the current disruption region, which has a larger spatial scale and a longer time scale, may have been the source current employed in the present paper.

\subsection{Conclusions}

The present paper investigated propagation of the MHD signal generated by the impulsive eastward current (the source current) as a simplified model of the Pi2 signal and Psc in the inner magnetosphere based on modal analysis using FEM. The compressional magnetic component in the plasmasphere was mainly investigated. The model has a dipole magnetic field with the ionosphere as the lower boundary of the magnetosphere. The outer boundary is placed far from the plasmasphere in order to eliminate the effect of the outer boundary from the behavior of the plasmasphere virtual resonance oscillation. The numerical calculation reveals the waveform of the plasmasphere virtual resonance oscillation of the Pi2 pulsations.

The following results were obtained:

1) The source current generates the plasmasphere virtual resonance oscillation. Evaluation of position and temporal variation of the source current may be possible. The source current is related to the large-scale magnetospheric eastward current that is associated with the current disruption.

2) When the source current is located in the outer magnetosphere $(L>15)$, the waveform becomes different from that of the typical Pi2 signal. Therefore, the source current that makes the typical Pi2 signal in the plasmasphere should be located fairly close to the plasmapause.

3) When the source current is distributed symmetrically with respect to the equator, only an azimuthal electric field and compressional magnetic field appear at the equator, whereas the poloidal magnetic field vanishes at the equator. When the current is located off-equator, the poloidal component appears at the equator and has a different waveform than that of the compressional component at the equator.

4) The compressional magnetic component appears to be confined within $\pm 20^{\circ} \sim 30^{\circ}$ around the magnetospheric equator in the plasmasphere. This is consistent with the result observed by the AKEBONO satellite (Osaki et al., 1998). 
The waveform of the impulse-stimulated pulsation (Pi2/Psc) is very important because it carries information on the source current. These pulsations have been studied based on spectral analysis. However, using pulsations such as the Pi2 pulsation in evaluating the source current properties requires the direct analysis of the waveform.

Acknowledgments. The authors are very grateful for discussions with K. Yumoto of Kyushu Univ. and T. Araki of Kyoto Univ. Constructive criticism from the referees helped to improve this paper. Numerical calculations were performed by computer centers of the Meteorological Research Institute and of the National Institute of Polar Research, as well as by the computer center of Nagoya University. This work was supported by the Grant-in-Aid for Scientific Research (c), No. 09640532, from The Ministry of Education, Science and Culture.

The Editor thanks D.-H. Lee and K. Takahashi for their assistance in evaluating this paper.

\section{Appendix A. Modal Analysis of the MHD Equation}

The basic equation for FEM eigenmode analysis is obtained as Eq. (3) without the r.h.s. term by multiplying $\delta \boldsymbol{E}_{\perp}^{*}$ (a piecewise FEM element function in Galerkin method) and then integrating the result in the magnetosphere. Galerkin formulation used in the present study is explained by Fujita and Patel (1992).

By assuming temporal variation as $e^{s t}$, the FEM equation is written as

$$
\begin{gathered}
\int_{m s} d V\left(\nabla \times \delta \boldsymbol{E}_{\perp}^{*}\right)\left(\nabla \times \delta \boldsymbol{E}_{\perp}\right)+\int_{m s} d V \frac{s^{2}}{V_{A}^{2}} \delta \boldsymbol{E}_{\perp}^{*} \delta \boldsymbol{E}_{\perp} \\
+\int_{i s} d S s \Sigma_{P} \delta \boldsymbol{E}_{\perp}^{*} \delta \boldsymbol{E}_{\perp}+\int_{m s} d V s \sigma \delta \boldsymbol{E}_{\perp}^{*} \delta \boldsymbol{E}_{\perp}=0 .
\end{gathered}
$$

(The ionospheric conductivity is included in Eq. (A.1) for generality, but is not considered in the present paper.) The eigenvectors obtained through Eq. (A.1) compose an orthogonal system. This means that any function can be expanded using these eigenvectors: this is the fundamental concept of modal analysis.

Discretizing Eq. (A.1) in space, we obtain

$$
s^{2} B \boldsymbol{X}+s C \boldsymbol{X}+A \boldsymbol{X}=0,
$$

where $\boldsymbol{X}$ is the row vector for FEM-discretized $\delta \boldsymbol{E}_{\perp} . A, B$, and $C$ are real matrices. Then, let $s_{i}$ and $\boldsymbol{z}_{i}$ be the eigenvalue and corresponding row eigenvector of Eq. (A.2), respectively. Note that $\bar{s}_{i}$ and $\bar{z}_{i}$ (complex conjugates) are also the eigenvalue and eigenvector of Eq. (A.2), respectively, because matrices $A, B$, and $C$ are real. The orthogonality of the eigenvector is defined as

$$
\begin{aligned}
& {\left[\begin{array}{ll}
s_{i} \boldsymbol{z}_{i}^{T} & \boldsymbol{z}_{i}^{T}
\end{array}\right]\left[\begin{array}{ll}
0 & B \\
B & C
\end{array}\right]\left[\begin{array}{c}
s_{j} \boldsymbol{z}_{j} \\
\boldsymbol{z}_{j}
\end{array}\right]} \\
& =\left(s_{j} \boldsymbol{z}_{i}^{T} B \boldsymbol{z}_{j}+s_{i} \boldsymbol{z}_{i}^{T} B \boldsymbol{z}_{j}+\boldsymbol{z}_{j}^{T} C \boldsymbol{z}_{i}\right) \delta_{i j}, \\
& {\left[\begin{array}{ll}
s_{i} \boldsymbol{z}_{i}^{T} & \boldsymbol{z}_{i}^{T}
\end{array}\right]\left[\begin{array}{cc}
B & 0 \\
0 & -A
\end{array}\right]\left[\begin{array}{c}
s_{j} \boldsymbol{z}_{j} \\
\boldsymbol{z}_{j}
\end{array}\right]} \\
& =\left(s_{j} s_{i} \boldsymbol{z}_{i}^{T} B \boldsymbol{z}_{j}-\boldsymbol{z}_{j}^{T} A \boldsymbol{z}_{i}\right) \delta_{i j},
\end{aligned}
$$

where $\boldsymbol{z}^{T}$ denotes the transposed vector of $\boldsymbol{z}$. These relations are valid for $\overline{\boldsymbol{z}}$.
Next, we explain the method used to obtain temporal variation of the MHD signal that is driven by an external source. Let $\boldsymbol{f}(t)$ be the FEM-discretized form of the source current $\left(\int d V \delta \boldsymbol{E}_{\perp}^{*}\left(-\mu_{0} \partial \boldsymbol{J} / \partial t\right)\right)$. By retaining time-derivatives in Eq. (A.1), the FEM-discretized form of Eq. (A.1) with the source current term in r.h.s. becomes

$$
B \ddot{\boldsymbol{X}}(t)+C \dot{\boldsymbol{X}}(t)+A \boldsymbol{X}(t)=\boldsymbol{f}(t) .
$$

When we define $\boldsymbol{Y}(t)$ as $\dot{\boldsymbol{X}}(t)$, Eq. (A.5) is transformed into

$$
\left[\begin{array}{ll}
0 & B \\
B & C
\end{array}\right]\left[\begin{array}{c}
\dot{\boldsymbol{Y}}(t) \\
\dot{\boldsymbol{X}}(t)
\end{array}\right]=\left[\begin{array}{cc}
B & 0 \\
0 & -A
\end{array}\right]\left[\begin{array}{c}
\boldsymbol{Y}(t) \\
\boldsymbol{X}(t)
\end{array}\right]+\left[\begin{array}{c}
0 \\
\boldsymbol{f}(t)
\end{array}\right]
$$

Next, we define

$$
\left[\begin{array}{l}
\boldsymbol{p}(t) \\
\boldsymbol{q}(t)
\end{array}\right]=\Phi^{-1}\left[\begin{array}{l}
\boldsymbol{Y}(t) \\
\boldsymbol{X}(t)
\end{array}\right]
$$

where

$$
\Phi=\left[\begin{array}{cc}
Z \Lambda & \bar{Z} \bar{\Lambda} \\
Z & \bar{Z}
\end{array}\right], \quad Z=\left[\boldsymbol{z}_{1} \cdots \boldsymbol{z}_{n}\right]
$$

where $n$ is the size of matrices, $A, B$, and $C . \Lambda$ is the diagonal matrix having the element $s_{i} \delta i j$. By using the orthogonal relation indicated by Eqs. (A.3) and (A.4), we obtain, after some algebraic manipulation, the following equations

$$
\begin{aligned}
& \alpha_{i} \dot{p}_{i}(t)=\beta_{i} p_{i}(t)+g_{i}(t), \\
& \bar{\alpha}_{i} \dot{q}_{i}(t)=\bar{\beta}_{i} q_{i}(t)+\bar{g}_{i}(t),
\end{aligned}
$$

where

$$
\begin{gathered}
\alpha_{i}=2 s_{i} \boldsymbol{z}_{i}^{T} B \boldsymbol{z}_{i}+\boldsymbol{z}_{i}^{T} C \boldsymbol{z}_{i}, \\
\beta_{i}=s_{i}^{2} \boldsymbol{z}_{i}^{T} B \boldsymbol{z}_{i}-\boldsymbol{z}_{i}^{T} C \boldsymbol{z}_{i},
\end{gathered}
$$

where $i=1, \ldots, n$. Note that the vector $\boldsymbol{g}(t)$ transports information about the source current, such as

$$
\boldsymbol{g}(t)=\left(g_{1}(t), \ldots, g_{n}(t)\right)^{T}=Z^{T} \boldsymbol{f}(t)
$$

This equation indicates that each $g_{i}(t)$ corresponds to the contribution of each eigenvector $\boldsymbol{z}_{i}$ to the source current $(\boldsymbol{f}(t))$. When Eq. (A.9) is solved for the initial condition that there are no disturbances at $t=0$, we obtain

$$
p_{i}(t)=e^{\left(\beta_{i} / \alpha_{i}\right) t}\left\{\int_{0}^{t} \frac{g_{i}\left(t^{\prime}\right)}{\alpha_{i}} e^{-\left(\beta_{i} / \alpha_{i}\right) t^{\prime}}\right\},
$$

and $q_{i}(t)=\bar{p}_{i}(t)$. Note that $\beta_{i} / \alpha_{i}=s_{i}$. Finally, the electric field perturbation $(\boldsymbol{X})$ is obtained as

$$
\begin{aligned}
\boldsymbol{X}(\boldsymbol{r}, t) & =Z(\boldsymbol{r}) \boldsymbol{p}(t)+\bar{Z}(\boldsymbol{r}) \boldsymbol{q}(t)=2 \Re Z(\boldsymbol{r}) \boldsymbol{p}(t) \\
& =2 \Re \sum_{i=1}^{n} \boldsymbol{z}_{i}(\boldsymbol{r}) p_{i}(t) .
\end{aligned}
$$

Equation (A.15) shows that $p_{i}(t)$ is a kind of "weight" of the eigenvector, $\boldsymbol{z}_{i}$ 


\section{References}

Allan, W., E. M. Poulter, and S. P. White, Hydromagnetic wave coupling in the magnetosphere-plasmapause effects on impulsive-excited resonances, Planet. Space Sci., 34, 1189-1200, 1986.

Fujita, S. and V. L. Patel, Eigenmode analysis of coupled magnetohydrodynamic oscillations in the magnetosphere, J. Geophys. Res., 97, 1377713788, 1992.

Fujita, S. and K.-H. Glassmeier, Magnetospheric cavity resonance oscillations with energy flow across the magnetopause, J. Geomag. Geoelectr., 47, 1277-1292, 1995.

Itonaga, M., A. Yoshikawa, and K. Yumoto, One-dimensional transient response of the inner magnetosphere at the magnetic equator, 1. Transfer function and poles, J. Geomag. Geoelectr., 49, 21-48, 1997a.

Itonaga, M., A. Yoshikawa, and K. Yumoto, One-dimensional transient response of the inner magnetosphere at the magnetic equator, 2. Analysis of waveform, J. Geomag. Geoelectr., 49, 49-68, 1997b.

Kivelson, M. G. and D. J. Southwood, Coupling of global magnetospheric MHD eigenmodes to field line resonances, J. Geophys. Res., 91, 43454351, 1986.

Lee, D.-H., Dynamics of MHD wave propagation in the low-latitude magnetosphere, J. Geophys. Res., 101, 15371-15386, 1996.

Lee, D.-H., On the generation mechanism of Pi 2 pulsations in the magnetosphere, Geophys. Res. Lett., 25, 583-586, 1998.

Lee, D.-H. and K. Kim, Compressional MHD waves in the magnetosphere: A new approach, J. Geophys. Res., 104, 12379-12385, 1999.

Lui, A. T. Y., Current disruption in the Earth's magnetosphere: Observations and models, J. Geophys. Res., 101, 13067-13088, 1996.

Ohtani, S., S. Kokubun and C. T. Russell, Radial expansion of the tail current disruption during substorms: Anew approach to the substorm onset region. J. Geophys. Res., 97, 3129-3136, 1992.

Osaki, H., K. Takahashi, H. Fukinishi, T. Nagatsuma, H. Oya, A. Matsuoka, and D. K. Milling, Pi2 pulsations observed from the Akebono satellite in the plasmasphere, J. Geophys. Res., 103, 17605-17615, 1998.

Pekrides, H., A. D. M. Walker, and P. R. Sutcliff, Global modeling of Pi 2 pulsations, J. Geophys. Res., 102, 14343-14354, 1997.

Samson, J. C., Pi 2 pulsations: High latitude results, Planet. Space Sci., 30, 1239-1247, 1982

Southwood, D. J. and M. G. Kivelson, The effect of parallel inhomogeneity on magnetospheric hydromagnetic wave coupling, J. Geophys. Res., 91, 6871-6876, 1986.

Sutcliffe, P. R. and K. Yumoto, Dayside Pi 2 pulsations at low latitudes, Geophys. Res. Lett., 16, 887-890, 1989.

Sutcliffe, P. R. and K. Yumoto, On the cavity mode nature of low-latitude Pi 2 pulsations, J. Geophys. Res., 96, 1543-1551, 1991.
Takahashi, K., Studies of magnetospheric ULF waves using Active Magnetospheric Particle Tracer Explorers Charge Composition Explorer, J. Geomag. Geoelectr., 46, 953-970, 1994.

Takahashi, K., L. J. Zanetti, R. E. Lopez, R. W. McEntire, T. A. Potemra, and K. Yumoto, Disruption of the magnetotail current sheet observed by AMPTE/CCE, Geophys. Res. Lett., 14, 1019-1022, 1987.

Takahashi, K., S.-I. Ohtani, and K. Yumoto, AMPTE CCE observations of Pi 2 pulsations in the inner magnetosphere, Geophys. Res. Lett., 19, 1447-1450, 1992.

Takahashi, K., S.-I. Ohtani, and B. J. Anderson, Statistical analysis of Pi2 pulsations observed by the AMPTE CCE spacecraft in the inner magnetosphere, J. Geophys. Res., 100, 21929-21941, 1995.

Takahashi, K., W. J. Hughes, R. R. Anderson, and S. I. Solovyev, CRRES satellite observations associated with low-latitude $\mathrm{Pi} 2$ pulsations, J. Geophys. Res., 104, 17431-17440, 1999.

Yeoman, T. K. and D. Orr, Phase and spectral power of mid-latitude Pi 2 pulsations: Evidence for a plasmaspheric cavity resonance, Planet. Space Sci., 37, 1367-1383, 1989.

Yumoto, K., Evidences of magnetospheric cavity Pi 2 waves, J. Geomag. Geoelectr., 42, 1281-1290, 1990.

Yumoto, K., K. Takahashi, T. Saito, F. W. Menk, B. J. Fraser, T. A. Potemra, and L. J. Zanetti, Some aspects of relation between Pi 1-2 magnetic pulsations observed at $L=1.3-2.1$ on the ground and substorm-associated magnetic field variations in the near-Earth magnetotail observed by AMPTE CCE, J. Geophys. Res., 94, 3611-3618, 1989.

Yumoto, K., K. Takahashi, T. Sakurai, P. R. Sutcliffe, S. Kokubun, H. Lühr, T. Saito, M. Kuwashima, and N. Sato, Multiple ground-based and satellite observations of global Pi 2 magnetic pulsations, J. Geophys. Res., 95, 15175-15184, 1990.

Yumoto, K., A. Isono, K. Shiokawa, H. Matsuoka, Y. Tanaka, F. W. Menk, B. J. Fraser, and $210^{\circ} \mathrm{MM}$ Magnetic Observation Group, Global cavity mode-like and localized field-line Pc3-4 oscillations stimulated by interplanetary impulses $(\mathrm{Si} / \mathrm{Sc})$ : Initial results from the $210^{\circ} \mathrm{MM}$ magnetic observations, in Solar Wind Sources of Magnetospheric Ultra-LowFrequency Waves, edited by M. J. Engebretson, K. Takahashi, and M. Scholer, 335 pp., American Geophysical Union, Washington, D.C., 1994

Yumoto, K., V. Pilipenko, E. Fedorov, N. Kurneva, M. De Lauretis, and K. Kitamura, Magnetospheric ULF wave phenomena stimulated by SSC, J. Geomag. Geoelectr., 49, 1179-1195, 1997.

S. Fujita (e-mail: sfujita@typhoon.mc-jma.ac.jp), H. Nakata (e-mail: nakata@kugi.kyoto-u.ac.jp), and M. Itonaga (e-mail: itonaga@po.cc. yamaguchi-u.ac.jp) 